\title{
An Asymptotic Preserving Scheme for the Diffusive Limit of Kinetic systems for Chemotaxis
}

\author{
José A. Carrillo ${ }^{1}$, Bokai Yan ${ }^{2}$ \\ ${ }^{1}$ Institució Catalana de Recerca i Estudis Avançats and Departament de Matemàtiques \\ Universitat Autònoma de Barcelona, E-08193 Bellaterra, Spain \\ Email: carrillo@mat.uab.es \\ On leave from: Department of Mathematics, Imperial College London, London SW7 2AZ, UK. \\ 2 Department of Mathematics \\ University of Wisconsin-Madison, WI 53706 \\ Email: yan@math.wisc.edu
}

November 21, 2018

\begin{abstract}
In this work we numerically study the diffusive limit of run \& tumble kinetic models for cell motion due to chemotaxis by means of asymptotic preserving schemes. It is well-known that the diffusive limit of these models leads to the classical Patlak-Keller-Segel macroscopic model for chemotaxis. We will show that the proposed scheme is able to accurately approximate the solutions before blow-up time for small parameter. Moreover, the numerical results indicate that the global solutions of the kinetic models stabilize for long times to steady states for all the analyzed parameter range. We also generalize these asymptotic preserving schemes to two dimensional kinetic models in the radial case. The blow-up of solutions is numerically investigated in all these cases.
\end{abstract}

\section{Introduction}

Chemotaxis is one of the basic mechanisms of cell motility due to chemical interaction. Cells are attracted by the concentration of certain chemical substance, called chemoattractant, and they direct their movement toward the regions of highest concentration. Typically, this phenomenon have been described based on macroscopic systems of equations describing the evolution of the cell density and the chemoattractant in time. These systems of drift-diffusion type are the well-know classical (Patlak)-Keller-Segel models [29, 24, 25]. Another point of view was introduced by a mesoscopic description of these phenomena bridging from stochastic interacting particle systems to macroscopic equations. This middle ground consists in describing the movement of cells by a "run \& tumble" process [26, 28]. The cells move along a straight line in the running phase and make reorientation as a reaction to the surrounding chemicals during the tumbling phase. This is the typical behavior that has been observed in experiments. The resulting nondimensionalized kinetic equation, with parabolic rescaling, reads

$$
\varepsilon \frac{\partial f}{\partial t}+v \cdot \nabla_{x} f=\frac{1}{\varepsilon} \int_{V}\left(T_{\varepsilon} f^{\prime}-T_{\varepsilon}^{*} f\right) \mathrm{d} v^{\prime}
$$

Here $f=f_{\varepsilon}(t, x, v)$ is the density of cells at position $x \in \mathbb{R}^{N}$, moving with velocity $v \in V \subset \mathbb{R}^{N} . S=S_{\varepsilon}(t, x)$ is the density of chemoattractant, whose governing equation will be described later. $T_{\varepsilon}=T_{\varepsilon}[S]\left(t, x, v, v^{\prime}\right)$ is 
the turning kernel operator. We use the abbreviation $f^{\prime}=f\left(t, x, v^{\prime}\right), T_{\varepsilon}^{*}[S]\left(t, x, v, v^{\prime}\right)=T_{\varepsilon}[S]\left(t, x, v^{\prime}, v\right)$. $\varepsilon$ is the ratio of the mean running length between jumps to the typical observation length scale. For simplicity we omit the dependence on $\varepsilon$ in notations (except $T_{\varepsilon}$, where $\varepsilon$ explicitly enters into the expression.) We refer to [11] for the details on the rescaling. The chemoattractant $S$ is typically given by the Poisson equation

$$
-\Delta S=\rho \quad \text { where } \rho=\rho_{\varepsilon}(t, x)=\int_{V} f_{\varepsilon}(t, x, v) \mathrm{d} v \text { is the macroscopic density of cells. }
$$

Here, we assume that the time scale of relaxation for the chemoattractant is much smaller that the one for the cell density. Although this is the most standard way of introducing the coupling with the chemoattractant, some authors have proposed to use some generalized models for any dimension where the chemoattractant $S$ is given by

$$
S=-\frac{1}{N \pi} \log |x| * \rho .
$$

Note that in two dimensional case, (1.3) is exactly (1.2).

Starting with the kinetic equation (1.1), one can (at lease formally) derive the macroscopic limit as $\varepsilon \rightarrow 0$

$$
\partial_{t} \rho=\nabla_{x} \cdot\left(D \nabla_{x} \rho-\chi \rho \nabla_{x} S\right) .
$$

The details will be described in Section 3 . Coupling with (1.2), one obtains the already mentioned PatlakKeller-Segel system [29, 24, 25] for chemotaxis. We refer to the reviews [17, 30] and the references therein for mathematical results on this system. The behavior of the solution is quite different depending on the dimension. In the $1 \mathrm{D}$ case, the global solution exists for all initial condition. In the $2 \mathrm{D}$ case, there exists a critical mass $M_{c}$ determined by the coefficients [13, 6]. The global solution exists if the initial total mass satisfies $M<M_{c}$ (subcritical case) and its long time asymptotics are given by self-similar profiles, otherwise the solution blows up in infinite (critical case $M=M_{c}[5]$ ) or finite time (supercritical case $M>M_{c}$ [14]). The critical case has infinitely many stationary states, each of them having their own basin of attraction [4] depending on the tail of the distribution at infinity. In the 3D case, the relevant quantity ensuring global existence is the $L^{3 / 2}$ norm of the initial density, see [12], together with some condition involving the second moment of the solution.

Similarly, starting with (1.1)-(1.3), one derives the modified Keller-Segel model (1.4)-(1.3) studied in [8], as $\varepsilon \rightarrow 0$. For the modified system, the existence of critical mass is extended to the $1 \mathrm{D}$ and $3 \mathrm{D}$ cases. It is given by

$$
M_{c}=\frac{2 N^{2} \pi D}{\chi}
$$

A very interesting consequence is that even in one dimension, this system blows-up over this critical mass. This makes possible to numerically analyse this highly subtle phenomena. This modified Keller-Segel system was numerically solved in [3] by using optimal transportation ideas and the scheme proven to be convergent in the subcritical case.

Alt in [1, 2] derived (1.4) from a transport equation similar to (1.1) via a stochastic model. Later the kinetic system (1.1)-(1.2) was formulated in [26]. Othmer and Hillen in [16, 27] studied the formal diffusion limit of this system by moment expansions. In [11] Chalub et al. proved that, in three dimensions with suitable assumptions on the turning kernel $T_{\varepsilon}$, the solution to the kinetic system (1.1)-(1.2) globally exists for any initial total mass. Moreover, they gave a rigorous proof of the convergence to the macroscopic limit for all time invervals of existence of the limiting Keller-Segel system. In the following work [18, 19] the results are extended to more general cases. 
The first task of this work is to design an Asymptotic-Preserving (AP) method for the kinetic system (1.1)-(1.3) in the one dimensional case. The "Asymptotic Preserving" scheme, introduced by [20], is a suitable numerical method for the kinetic equation in such a way that letting $\varepsilon \rightarrow 0$ with the mesh size and time step fixed, the scheme becomes a scheme for the limiting system. In this case, this means as $\varepsilon \rightarrow 0$, the moments of the solution to the kinetic system (1.1)-(1.3) solve the modified Keller-Segel system (1.4)-(1.3) automatically. We refer to [21] and the references therein for a detailed review on AP scheme.

As mentioned above, the solution to the modified Keller-Segel system (1.4)-(1.3) over the critical mass blows up in finite time $t^{*}$. But the global solution to the kinetic system (1.1)-(1.3) exists. An attractive idea is that, the solution of the kinetic system after $t^{*}$ might be a good candidate to describe the behavior of solution to the Keller-Segel system after blow-up. There are other mechanisms of regularizing the PatlakKeller-Segel system after blow-up by saturating the effect of the chemoattractant, introducing volume effects or cross-diffusion in the macroscopic system, see [10] for a discussion. However, to regularize it by the kinetic system is more appealing since the kinetic system is directly derived from interacting particle systems models.

Bournaveas and Calvez [7] studied the kinetic system (1.1)-(1.2) with the local turning kernel $T_{\varepsilon}$ defined below in (2.6). They showed that, in the two dimensional spherically symmetric coordinate, the solution exists below a critical mass $m_{c}$ and blows up over a critical mass $M_{c}$. Two questions remains unsolved. First, the large-time behavior of the solutions for subcritical cases is not clear yet. Second, the two thresholds do not match, i.e. $m_{c}<M_{c}$. Moreover, the appearance of blow-up in the one dimensional case has not been clarified yet, see [31]. The second aim of this work is to investigate these questions through numerical simulation.

The outline of the paper is as follows. In section 2 the kinetic models we will work on are described. Section 3 gives the macroscopic limit of each model. Section 4 describes the AP schemes for these models. Finally, in section 5 we present the simulation experiments with our schemes and draw some conclusions.

\section{The kinetic models}

In this section we briefly describe the kinetic models we used in our simulations. The chemoattractant $S$ is always given by the log kernel convolution (1.3).

The turning kernel $T_{\varepsilon}$ in the kinetic equation (1.1) needs to be specified. The turning kernel, $T_{\varepsilon}=$ $T_{\varepsilon}[S]\left(t, x, v, v^{\prime}\right) \geq 0$, measures the probability of velocity jump of cells from $v^{\prime}$ to $v$. To derive the KellerSegel equation (1.4) as $\varepsilon \rightarrow 0$, one has to incorporate both $O(1)$ and $O(\varepsilon)$ scale into $T_{\varepsilon}$. In the following work, we consider $T_{\varepsilon}$ in the form

$$
T_{\mathcal{E}}[S]\left(t, x, v, v^{\prime}\right)=F(v)+\varepsilon T_{1}+O\left(\varepsilon^{2}\right) .
$$

Here $F(v) \geq 0$ gives the equilibrium of velocity distribution when there is no directional preference. It is natural to assume

$$
\left\{\begin{array}{l}
\int_{V} F(v) \mathrm{d} v=1 \\
F(v)=F(|v|)
\end{array} .\right.
$$

$T_{1}$ characterizes the directional preference. We can assume $T_{1} \geq 0$ since we are considering the reaction of cells to chemoattractant. The cells have a larger probability to jump to a preferred direction. 


\section{$2.11 D$ nonlocal Model}

Now we employ the nonlinear kernel introduced in [11],

$$
T_{\varepsilon}=T_{\varepsilon}[S]\left(t, x, v, v^{\prime}\right)=\alpha_{+} \psi(S(t, x), S(t, x+\varepsilon v))+\alpha_{-} \psi\left(S(t, x), S\left(t, x-\varepsilon v^{\prime}\right)\right) .
$$

The first term means the cell decides a new direction to move based on the detection of current environment and probable new location, while the second term gives the influence of past memory on the choice of the new direction. For simplicity we neglect the second term. We take $\alpha_{+}=1, \alpha_{-}=0$, and we consider

$$
\psi(S, \tilde{S})=F(v)+(\tilde{S}-S)_{+}
$$

Again $F=F(|v|)$ is the equilibrium function satisfying (2.1). For simplicity we introduce the notation

$$
\delta^{\varepsilon} S(x, v)=(S(x+\varepsilon v)-S(x))_{+} .
$$

Note that $\delta^{\varepsilon} S=O(\varepsilon)$. Then the kinetic equation (1.1) reads

$$
\varepsilon \frac{\partial f}{\partial t}+v \frac{\partial f}{\partial x}=\frac{1}{\varepsilon}\left(\left(F(v)+\delta^{\varepsilon} S(x, v)\right) \rho-\left(1+\int \delta^{\varepsilon} S\left(x, v^{\prime}\right) \mathrm{d} v^{\prime}\right) f\right)
$$

where $x \in \Omega=\left[-x_{\max }, x_{\max }\right], v \in V=\left[-v_{\max }, v_{\max }\right],|V|=\operatorname{Vol}(V)$. We impose the initial conditions,

$$
\begin{gathered}
f(0, x, v,)=f^{I}(x, v) \geq 0, \\
S(0, x)=S^{I}(x) \geq 0,
\end{gathered}
$$

and reflection boundary condition for $f$, Neumann boundary condition for $S$,

$$
\begin{aligned}
f\left(t, \pm x_{\max }, v\right) & =f\left(t, \pm x_{\max },-v\right), \\
\left.\partial_{x} S\right|_{x= \pm x_{\max }} & =0 .
\end{aligned}
$$

Equations (2.3)-(1.3) gives the nonlocal model in one dimension. The global solution exists, regardless of the total mass.

\section{2 $1 D$ local Model}

In this section we summarize a turning kernel introduced by Bournaveas and Calvez [7]. Let

$$
T_{\varepsilon}=T_{\varepsilon}[S]\left(t, x, v, v^{\prime}\right)=F(v)+\varepsilon(v \cdot \nabla S(x))_{+},
$$

where $F=F(|v|)$ is the equilibrium function satisfying (2.1). Then the kinetic equation (1.1) in one dimension reads

$$
\varepsilon \frac{\partial f}{\partial t}+v \frac{\partial f}{\partial x}=\frac{1}{\varepsilon}\left(\left(F(v)+\varepsilon(v \cdot \nabla S)_{+}\right) \rho-\left(1+c_{1} \varepsilon|\nabla S|\right) f\right)
$$

with $c_{1}=\int_{V}(v \cdot \nabla S /|\nabla S|)_{+} \mathrm{d} v=\frac{1}{2} \int_{V}|v| \mathrm{d} v$. The same initial conditions (2.4a) (2.4b), and boundary conditions (2.5a), 2.5b) are applied.

Remark 2.1. If we expand the kernel (2.2) $T=T_{0}+\varepsilon T_{1}+O\left(\varepsilon^{2}\right)$, and drop the terms higher than first order, we get exactly the kernel (2.6). 


\subsection{D local Model}

Plug the local kernel (2.6) into the kinetic equation (1.1) in 2D, one obtains,

$$
\left\{\begin{array}{l}
\varepsilon \partial_{t} f+v \cdot \nabla_{x} f=\frac{1}{\varepsilon}\left(\rho F(v)-f_{\varepsilon}+\varepsilon \rho(v \cdot \nabla S)_{+}-\varepsilon c_{2}|\nabla S| f\right), \quad v \in V=B\left(0, v_{\max }\right), x \in \mathbb{R}^{2} \\
-\nabla^{2} S=\rho
\end{array}\right.
$$

with $c_{2}=\int_{V}(v \cdot \nabla S /|\nabla S|)_{+} \mathrm{d} v$. The equilibrium $F(v)$ satisfies (2.1).

Now we assume the initial data $f^{I}$ is spherically symmetric: $f^{I}(\Theta x, \Theta v)=f^{I}(x, v)$, for any rotation $\Theta$. Then the solution $f$ to 2.8 remains spherically symmetric. Denote

$$
r=|x| \in[0, \infty), \quad \omega=|v| \in\left[0, v_{\max }\right), \quad \theta=\cos ^{-1} \frac{x \cdot v}{r \omega} \in[0, \pi] .
$$

Then $f$ is a function of $r, \omega, \theta$ and $t$. Let

$$
\begin{gathered}
h(t, r, \omega, \theta)=r f\left(t,\left.x\right|_{(r, 0)},\left.v\right|_{(\omega \cos \theta, \omega \sin \theta)}\right)=r f\left(t,\left.x\right|_{(r \cos \theta, r \sin \theta)},\left.v\right|_{(\omega, 0)}\right), \\
\tilde{\rho}(t, r)=r \rho(t, r) .
\end{gathered}
$$

Then the density is given by

$$
\tilde{\rho}(t, r)=r \rho(t, r)=r \int_{V} f(t,|x|=r, v) \mathrm{d} v=2 \iint_{0 \leq \omega \leq v_{\max }, 0 \leq \theta \leq \pi} \omega h \mathrm{~d} \theta \mathrm{d} \omega,
$$

and the total mass is

$$
M=2 \pi \int_{0}^{\infty} \tilde{\rho}(t, r) \mathrm{d} r=4 \pi \iiint_{0 \leq \omega \leq v_{\max }, 0 \leq \theta \leq \pi, 0 \leq r} \omega h \mathrm{~d} \theta \mathrm{d} \omega \mathrm{d} r .
$$

Then equation (2.8) can be rewritten as

$$
\left\{\begin{array}{l}
\partial_{t} h+\frac{\omega}{\varepsilon}\left(\partial_{r}(\cos \theta h)-\partial_{\theta}\left(\frac{\sin \theta}{r} h\right)\right)=\frac{1}{\varepsilon^{2}}(\tilde{\rho} F(\omega)-h)+\frac{1}{\varepsilon}\left(\omega \tilde{\rho}\left(\cos \theta \partial_{r} S\right)_{+}-c_{2}\left|\partial_{r} S\right| h\right), \\
-\partial_{r}\left(r \partial_{r} S\right)=\tilde{\rho}
\end{array}\right.
$$

where

$$
c_{2}=2 \int_{0}^{\pi} \int_{0}^{v_{\max }} \omega^{2}\left(\cos \theta \partial_{r} S /\left|\partial_{r} S\right|\right)_{+} \mathrm{d} \omega \mathrm{d} \theta=\int_{0}^{\pi} \int_{0}^{v_{\max }} \omega^{2}|\cos \theta| \mathrm{d} \omega \mathrm{d} \theta=\frac{2 v_{\max }^{3}}{3},
$$

and $F(\omega)$ satisfies,

$$
2 \pi \int_{0}^{v_{\max }} \omega F(\omega) \mathrm{d} \omega=1 .
$$

From the second equation of (2.9), $\partial_{r} S$ can be computed by

$$
\partial_{r} S=-\frac{1}{r} \int_{0}^{r} \tilde{\rho} \mathrm{d} r
$$

We impose the boundary conditions,

$$
\begin{aligned}
h(0, \omega, \theta) & =h(0, \omega, \pi-\theta), \\
\partial_{r} h\left(r_{\max }, \omega, \theta\right) & =0 .
\end{aligned}
$$

It has been shown that the solution blows up in finite time in [7] for $M$ large enough while existing globally for small enough mass. 


\section{The macroscopic limits}

\subsection{D model: local and nonlocal}

The local kinetic model (2.7) and the nonlocal kinetic model (2.3) give the same asymptotic limit as $\varepsilon \rightarrow 0$. We apply the Hilbert expansion into (2.7) and (2.3), and match terms of the same order in $\varepsilon$. The classical Keller-Segel system can be derived for $\rho(t, x)=\int_{V} f(t, x, v) \mathrm{d} v$,

$$
\partial_{t} \rho=\partial_{x}\left(D \partial_{x} \rho-\chi \rho \partial_{x} S\right)
$$

where

$$
D=\int_{V}|v|^{2} F(v) \mathrm{d} v, \quad \text { and } \quad \chi=\frac{1}{2} \int_{V}|v|^{2} \mathrm{~d} v .
$$

We refer to [11] for the details. Besides, by taking the moments of the kinetic equation (1.1), one has

$$
\partial_{t} \rho+\nabla_{x} \cdot\left(\frac{1}{\varepsilon} \int_{V} v f \mathrm{~d} v\right)=0 .
$$

Compare with 3.1 , one has

$$
D \partial_{x} \rho-\chi \rho \partial_{x} S=-\frac{1}{\varepsilon} \int_{V} v f \mathrm{~d} v .
$$

While the reflection boundary condition (2.5a) leads to

$$
\int_{V} v f \mathrm{~d} v=0, \quad \text { at } x= \pm x_{\max } .
$$

One arrives at the general boundary condition (for mass preservation) for Keller-Segel model

$$
D \partial_{x} \rho-\chi \rho \partial_{x} S=0, \quad \text { at } x= \pm x_{\max } .
$$

Furthermore, the Neumann boundary condition for $\rho$ is derived under the condition $(2.5 \mathrm{~b})$.

\subsection{D spherical symmetric local model}

For the reduced spherical symmetric kinetic system (2.9), one can derive the similar asymptotic limit for $\tilde{\rho}$ as $\varepsilon \rightarrow 0$,

$$
\partial_{t} \tilde{\rho}=\partial_{r}\left(\operatorname{Dr} \partial_{r} \frac{\tilde{\rho}}{r}-\chi \tilde{\rho} \partial_{r} S\right)
$$

with

$$
-\partial_{r}\left(r \partial_{r} S\right)=\tilde{\rho}
$$

where $r \in\left[0, r_{\max }\right]$ with

$$
D=2 \int_{0}^{\pi} \int_{0}^{v_{\max }} \omega^{3} \cos ^{2} \theta F(\omega) \mathrm{d} \omega \mathrm{d} \theta=\pi \int_{0}^{v_{\max }} \omega^{3} F(\omega) \mathrm{d} \omega
$$

and

$$
\chi=\int_{0}^{\pi} \int_{0}^{v_{\max }} \omega^{3} \cos ^{2} \theta \mathrm{d} \omega \mathrm{d} \theta=\frac{\pi v_{\max }^{4}}{8} .
$$

The Neumann boundary condition is derived,

$$
\begin{aligned}
& \partial_{r} S=0, \quad \text { at } r=0, r_{\max }, \\
& \partial_{r} \frac{\tilde{\rho}}{r}=0, \quad \text { at } r=r_{\max } .
\end{aligned}
$$




\section{The Numerical Scheme}

In this section we present the numerical method for the kinetic system $(2.3)-(2.5 b)$ by the even and odd parity formalism, which has been successfully applied to the diffusive limit of linear transport equations in [23].

\subsection{Odd and Even Parity}

We introduce the operator

$$
\begin{aligned}
& R[f](x, v)=\frac{1}{2}(f(x, v)+f(x,-v)), \\
& J[f](x, v)=\frac{1}{2 \varepsilon}(f(x, v)-f(x,-v)) .
\end{aligned}
$$

The new functions $R[f]$ and $J[f]$ are defined in $\Omega \times V^{+} \times \mathbb{R}_{+}$, where $V^{+}=\{v \in V \mid v \geq 0\}$. Then we take

$$
r(x, v)=R[f]=\frac{1}{2}(f(x, v)+f(x,-v)), \quad j(x, v)=J[f]=\frac{1}{2 \varepsilon}(f(x, v)-f(x,-v)) .
$$

We can recover $f$ from $r$ and $j$,

$$
f(x, v)= \begin{cases}r(x, v)+\varepsilon j(x, v), & \text { if } v \geq 0 \\ r(x,-v)-\varepsilon j(x,-v), & \text { if } v<0 .\end{cases}
$$

\subsubsection{D nonlocal model}

Now we describe the odd and even decomposition for the nonlocal model. Since $f(x,-v)$ satisfies

$$
\varepsilon \frac{\partial f(x,-v)}{\partial t}-v \frac{\partial f(x,-v)}{\partial x}=\frac{1}{\varepsilon}\left(\left(F(v)+\delta^{\varepsilon} S(x,-v)\right) \rho-\left(1+\int \delta^{\varepsilon} S\left(x, v^{\prime}\right) \mathrm{d} v^{\prime}\right) f(x,-v)\right),
$$

we obtain,

$$
\begin{aligned}
\partial_{t} r+v \partial_{x} j & =\frac{1}{\varepsilon^{2}}\left(\left(F(v)+R\left[\delta^{\varepsilon} S\right]\right) \rho-\left(1+<\delta^{\varepsilon} S>\right) r\right), \\
\partial_{t} j+\frac{1}{\varepsilon^{2}} v \partial_{x} r & =\frac{1}{\varepsilon^{2}}\left(J\left[\delta^{\varepsilon} S\right] \rho-\left(1+<\delta^{\varepsilon} S>\right) j\right),
\end{aligned}
$$

where $<\delta^{\varepsilon} S>=\int \delta^{\varepsilon} S\left(x, v^{\prime}\right) \mathrm{d} v^{\prime}$, and

$$
\left.\rho=\int_{V} f \mathrm{~d} v=\int_{V^{+}}(f(x, v)+f(x,-v))\right) \mathrm{d} v=2 \int_{V^{+}} r(x, v) \mathrm{d} v .
$$

We assume $\varepsilon \leq 1$, then we can rewrite the equations for $r$ and $j$ as,

$$
\begin{aligned}
& \partial_{t} r+v \partial_{x} j=\frac{1}{\varepsilon^{2}}\left(\left(F(v)+R\left[\delta^{\varepsilon} S\right]\right) \rho-\left(1+<\delta^{\varepsilon} S>\right) r\right), \\
& \partial_{t} j+v \partial_{x} r=\frac{1}{\varepsilon^{2}}\left(J\left[\delta^{\varepsilon} S\right] \rho-\left(1+<\delta^{\varepsilon} S>\right) j\right)+\left(1-\frac{1}{\varepsilon^{2}}\right) v \partial_{x} r .
\end{aligned}
$$

Let us finish with the boundary conditions for new variables $r$ and $j$. From the reflection boundary condition 2.5a) and the definition of $r$ and $j$, we can easily get the boundary condition for $j$,

$$
j\left(t, x_{b}, v\right)=\frac{1}{2 \varepsilon}\left(f\left(t, x_{b}, v\right)-f\left(t, x_{b},-v\right)\right)=0 .
$$


where $x_{b}= \pm x_{\max }$. Then plug this into the second equation of (4.2), we derive,

$$
\left.v \partial_{x} r\right|_{x=x_{b}}=\left.J\left[\delta^{\varepsilon} S\right] \rho\right|_{x=x_{b}}=\frac{1}{2 \varepsilon}\left(\delta^{\varepsilon} S\left(x_{b}, v\right)-\delta^{\varepsilon} S\left(x_{b},-v\right)\right) \rho .
$$

Now from the Neumann boundary condition (2.5b), we have $S\left(x_{b}+\varepsilon v\right)=S\left(x_{b}-\varepsilon v\right)$, i.e. $\delta^{\varepsilon} S\left(x_{b}, v\right)=$ $\delta^{\varepsilon} S\left(x_{b},-v\right)$. So the boundary condition for $r$ is

$$
\left.\partial_{x} r\right|_{x=x_{b}}=0
$$

Now, we can propose an asymptotic preserving method for the one dimensional nonlocal model. The idea can be applied to the other models straightforwardly.

As $\varepsilon$ approaches 0 , we can derive the Keller-Segel equation (1.4) asymptotically from the kinetic equation (2.3). So a natural requirement is the numerical method for (2.3) should discretize its macroscopic limit as $\varepsilon \rightarrow 0$. We give an AP method following [22].

We can employ an operator splitting method on (4.2). First the (stiff) source part is solved by the implicit Euler method,

$$
\begin{aligned}
& \partial_{t} r=\frac{1}{\varepsilon^{2}}\left(\left(F(v)+R\left[\delta^{\varepsilon} S\right]\right) \rho-\left(1+<\delta^{\varepsilon} S>\right) r\right), \\
& \partial_{t} j=\frac{1}{\varepsilon^{2}}\left(J\left[\delta^{\varepsilon} S\right] \rho-\left(1+<\delta^{\varepsilon} S>\right) j\right)+\left(1-\frac{1}{\varepsilon^{2}}\right) v \partial_{x} r .
\end{aligned}
$$

Then the transport part can be solved by an explicit method (e.g. upwind scheme),

$$
\begin{aligned}
& \partial_{t} r+v \partial_{x} j=0, \\
& \partial_{t} j+v \partial_{x} r=0 .
\end{aligned}
$$

We can check that the method described above is AP easily. As $\varepsilon \rightarrow 0$ the leading term in $\varepsilon$ of (4.5) gives,

$$
\begin{aligned}
& r=\frac{F(v)+R\left[\delta^{\varepsilon} S\right]}{1+<\delta^{\varepsilon} S>} \rho=\rho F(v)+O(\varepsilon), \\
& j=\frac{J\left[\delta^{\varepsilon} S\right] \rho-v \partial_{x} r}{1+<\delta^{\varepsilon} S>}=v\left(\frac{1}{2} \partial_{x} S \rho-\partial_{x} r\right)+O(\varepsilon) .
\end{aligned}
$$

Plug into the first equation of (4.6) and integrate over $V^{+}$, we get exactly the Keller-Segel equation (1.4) with $D$ and $\chi$ given by (3.2).

\subsubsection{D local model}

For the one dimensional local model, we obtain,

$$
\begin{aligned}
\partial_{t} r+v \partial_{x} j & =\frac{1}{\varepsilon^{2}}\left(\left(F(v)+\frac{\varepsilon}{2}\left|v \partial_{x} S\right|\right) \rho-\left(1+c_{1} \varepsilon\left|\partial_{x} S\right|\right) r\right), \\
\partial_{t} j+\frac{1}{\varepsilon^{2}} v \partial_{x} r & =\frac{1}{\varepsilon^{2}}\left(\frac{1}{2} v \partial_{x} S \rho-\left(1+c_{1} \varepsilon\left|\partial_{x} S\right|\right) j\right) .
\end{aligned}
$$

The remaining work is similar to the nonlocal model. The boundary conditions for for new variables $r$ and $j$ are also given by (4.4) and (4.3). The AP scheme can be designed similarly. 


\subsubsection{D spherical symmetric local model}

Denote

$$
R=\frac{1}{2}(h(r, \omega, \theta)+h(r, \omega, \pi-\theta)), \quad J=\frac{1}{2 \varepsilon}(h(r, \omega, \theta)-h(r, \omega, \pi-\theta)) .
$$

$R$ and $J$ are functions defined on $\left[0, r_{\max }\right] \times\left[0, v_{\max }\right] \times[0, \pi]$. Then we can write the equations for $R$ and $J$,

$$
\begin{aligned}
\partial_{t} R+\omega\left(\partial_{r}(\cos \theta J)-\partial_{\theta}\left(\frac{\sin \theta}{r} J\right)\right) & =\frac{1}{\varepsilon^{2}}(\tilde{\rho} F-R)+\frac{1}{\varepsilon}\left(\frac{1}{2} \omega \tilde{\rho} \cos \theta\left|\partial_{r} S\right|-c_{2}\left|\partial_{r} S\right| R\right), \\
\partial_{t} J+\frac{\omega}{\varepsilon^{2}}\left(\partial_{r}(\cos \theta R)-\partial_{\theta}\left(\frac{\sin \theta}{r} R\right)\right) & =-\frac{1}{\varepsilon^{2}} J+\frac{1}{\varepsilon}\left(\frac{1}{2 \varepsilon} \omega \tilde{\rho} \cos \theta \partial_{r} S-c_{2}\left|\partial_{r} S\right| J\right) .
\end{aligned}
$$

Now we split this into two steps. First the collision step can be solved implicitly,

$$
\begin{aligned}
& \partial_{t} R=\frac{1}{\varepsilon^{2}}(\tilde{\rho} F-R)+\frac{1}{\varepsilon}\left(\frac{1}{2} \omega \tilde{\rho} \cos \theta\left|\partial_{r} S\right|-c_{2}\left|\partial_{r} S\right| R\right) \\
& \partial_{t} J=-\frac{1}{\varepsilon^{2}} J+\frac{1}{\varepsilon}\left(\frac{1}{2 \varepsilon} \omega \tilde{\rho} \cos \theta \partial_{r} S-c_{2}\left|\partial_{r} S\right| J\right)+\left(1-\frac{1}{\varepsilon^{2}}\right) \omega\left(\partial_{r}(\cos \theta R)-\partial_{\theta}\left(\frac{\sin \theta}{r} R\right)\right) .
\end{aligned}
$$

Then the transport part can be solved explicitly,

$$
\begin{aligned}
& \partial_{t} R+\omega\left(\partial_{r}(\cos \theta J)-\partial_{\theta}\left(\frac{\sin \theta}{r} J\right)\right)=0, \\
& \partial_{t} J+\omega\left(\partial_{r}(\cos \theta R)-\partial_{\theta}\left(\frac{\sin \theta}{r} R\right)\right)=0 .
\end{aligned}
$$

One can show $\tilde{\rho}$ solves the macroscopic limit (3.3) as $\varepsilon \rightarrow 0$.

\subsection{Time and space discretization}

\subsubsection{D system: nonlocal and local}

In this section we give full discretization for the 1D nonlocal kinetic system. The discretization for the 1D local system is similar. First we solve (4.5) by implicit Euler method,

$$
\begin{aligned}
& \frac{r^{*}-r^{n}}{\Delta t}=\frac{1}{\varepsilon^{2}}\left(\left(F(v)+R\left[\delta^{\varepsilon} S^{*}\right]\right) \rho^{*}-\left(1+<\delta^{\varepsilon} S^{*}>\right) r^{*}\right) \\
& \frac{j^{*}-j^{n}}{\Delta t}=\frac{1}{\varepsilon^{2}}\left(J\left[\delta^{\varepsilon} S^{*}\right] \rho^{*}-\left(1+<\delta^{\varepsilon} S^{*}>\right) j^{*}\right)+\left(1-\frac{1}{\varepsilon^{2}}\right) v \partial_{x}^{(c)} r^{*}
\end{aligned}
$$

where $r^{n}$ and $j^{n}$ are the numerical solutions of $r$ and $j$ at time $t_{n} . \partial_{x}^{(c)}$ is the central difference discretization of $\partial_{x}$. And a linear interpolation is used to get $S(x+\varepsilon v)$ and $S(x-\varepsilon v) .<\delta^{\varepsilon} S>$ is evaluated by applying the trapezoidal rule on the interpolated value.

If we integrate (4.5) over $V^{+}$, we can get $\partial_{t} \rho=0$. So

$$
\rho^{*}=\rho^{n}, \quad S^{*}=S^{n} .
$$


Therefore $r^{*}$ and $j^{*}$ can be solved explicitly,

$$
\begin{aligned}
& r^{*}=\frac{\varepsilon^{2} r^{n}+\Delta t\left(F(v)+R\left[\delta^{\varepsilon} S^{n}\right]\right) \rho^{n}}{\varepsilon^{2}+\Delta t\left(1+<\delta^{\varepsilon} S^{n}>\right)} \\
& j^{*}=\frac{\varepsilon^{2} j^{n}+\Delta t\left(J\left[\delta^{\varepsilon} S^{n}\right] \rho^{n}+\left(\varepsilon^{2}-1\right) v \partial_{x}^{(c)} r^{*}\right)}{\varepsilon^{2}+\Delta t\left(1+<\delta^{\varepsilon} S^{n}>\right)} .
\end{aligned}
$$

Then we apply a first-order upwind scheme on (4.6) to get $r^{n+1}, j^{n+1}$,

$$
\begin{aligned}
& \frac{r_{i}^{n+1}-r_{i}^{n}}{\Delta t}+\frac{v}{2 \Delta x}\left(j_{i+1}-j_{i-1}\right)=\frac{v}{2 \Delta x}\left(r_{i+1}-2 r_{i}+r_{i-1}\right), \\
& \frac{j_{i}^{n+1}-j_{i}^{n}}{\Delta t}+\frac{v}{2 \Delta x}\left(r_{i+1}-r_{i-1}\right)=\frac{v}{2 \Delta x}\left(j_{i+1}-2 j_{i}+j_{i-1}\right) .
\end{aligned}
$$

As $\varepsilon \rightarrow 0$, a simple computation shows that this scheme leads to (after integration over $V^{+}$),

$$
\frac{\rho^{n+1}-\rho^{n}}{\Delta t}=\partial_{x}^{(c)}\left(D \partial_{x}^{(c)} \rho^{n}-\chi \rho^{n} \partial_{x}^{(c)} S^{n}\right)+\Delta x C(V) \partial_{x x}^{(c)} \rho^{n} .
$$

with $D$ and $\chi$ given by (3.2), $\rho$ given by (4.1). Here $\partial_{x x}^{(c)}$ is the general three-point central difference of $\partial_{x x} . C(V)$ is a constant which only depends on the velocity space $V$. In the case of $V=\left[-v_{\max }, v_{\max }\right]$, $C(V)=v_{\max } / 4$.

\subsubsection{D spherical symmetric local model}

We describe a first order discretization for the transport part (4.7) of spherical symmetric system. By introducing

$$
P(x, v)=\frac{1}{2}(R(x, v)+J(x, v)), \quad Q(x, v)=\frac{1}{2}(R(x, v)-J(x, v)),
$$

one obtains

$$
\begin{gathered}
\partial_{t} P+\omega\left(\partial_{r}(\cos \theta P)-\partial_{\theta}\left(\frac{\sin \theta}{r} P\right)\right)=0 \\
\partial_{t} Q-\omega\left(\partial_{r}(\cos \theta Q)-\partial_{\theta}\left(\frac{\sin \theta}{r} Q\right)\right)=0
\end{gathered}
$$

We take the grid points at

$$
\begin{aligned}
r_{i} & =\left(i-\frac{1}{2}\right) \Delta r, \\
\theta_{j} & =\left(j-\frac{1}{2}\right) \Delta \theta, \quad j=1, \ldots, N,
\end{aligned}
$$

The grid points and the characteristic lines are shown in Figure1 We can define the flux at each interface according to the "upwind" value along the characteristic direction. An analogous idea was successfully used in the case of numerical simulation of the Boltzmann-Poisson system for semiconductors in [9]. Here we give the detailed discretization for $P$. The discretization for $Q$ is similar. For each $\omega$,

$$
\frac{P_{i, j}^{n+1}-P_{i, j}^{n}}{\Delta t}+\omega\left(\frac{F_{i+1 / 2, j}^{n}-F_{i-1 / 2, j}^{n}}{\Delta r}+\frac{G_{i, j+1 / 2}^{n}-G_{i, j-1 / 2}^{n}}{\Delta \theta}\right)=0,
$$




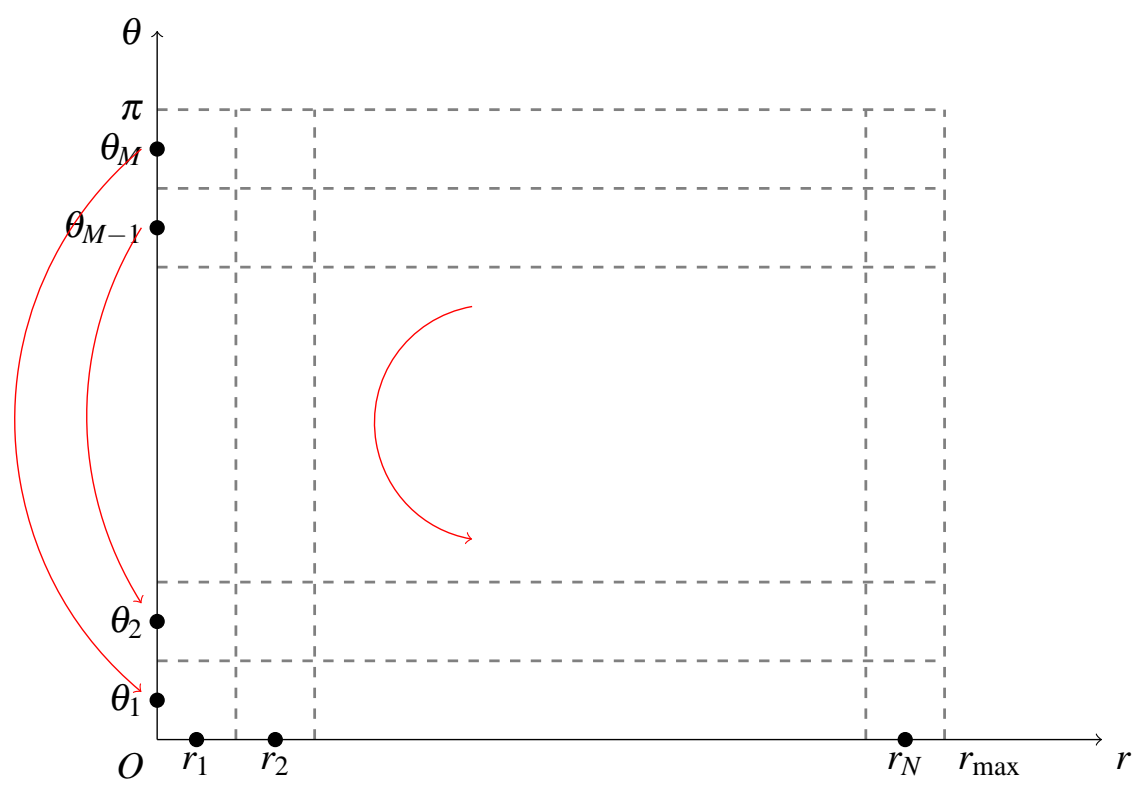

Figure 1: Illustration of grid points and characteristic lines on $r-\theta$ plane.

where

$$
\begin{gathered}
F_{i+1 / 2, j}^{n}=\left\{\begin{array}{lc}
\left(\cos \theta_{j}\right) P_{i, j}^{n}, & \text { if } j \leq M / 2, \\
\left(\cos \theta_{j}\right) P_{i+1, j}^{n}, & \text { if } j \geq M / 2+1,
\end{array}\right. \\
G_{i, j+1 / 2}^{n}=-\frac{\sin \left(\theta_{j}+\Delta \theta / 2\right)}{r_{i}} P_{i, j}^{n}=-\frac{\sin (j \Delta \theta)}{r_{i}} P_{i, j}^{n},
\end{gathered}
$$

with boundary

$$
F_{1 / 2, j}^{n}=F_{1 / 2, M-j}^{n}=\left(\cos \theta_{M-j}\right) P_{1, M-j}^{n}, \text { if } j \leq M / 2 .
$$

\subsection{Adaptive grids for solutions with blowup}

Now let us consider the case that the initial total mass is large. It has been shown that the solutions to the 2D spherical symmetric kinetic model (2.9) blow up in finite time. While for the one dimensional kinetic local model (2.7), although the strict analysis is lacking [31], the numerical results strongly suggest that the solutions also blow up. In these cases, the convergence of the schemes described above would be questionable when simulations are performed on the fixed grids. See section for the verification of convergence order.

Noting that in the blowup case, the magnitude of $\nabla_{x} S$ grows up dramatically as time evolves, which actually characterizes another scale for the kinetic equation. To capture this scale, one has to take $\Delta x \sim$ $\frac{1}{\max \left|\nabla_{X} S\right|}$. Hence the adaptive grids are needed. In numerical simulation, we double the grid points once $\left\|\nabla_{x} S\right\|_{\infty}$ is doubled. More exactly, we apply the following algorithm.

Algorithm 4.1. (Adaptive grids)

$$
\begin{aligned}
& s=\left\|\nabla_{x} S_{0}\right\|_{\infty} \\
& \text { if }\left\|\nabla_{x} S_{n}\right\|_{\infty} \geq 2 s \text { then } \\
& \quad s \leftarrow\left\|\nabla_{x} S_{n}\right\|_{\infty} .
\end{aligned}
$$


Double the grids, half the time step size $\Delta t$.

Derive $f^{n}$ on the finer grids by interpolation and continue the time evolution.

\section{end if}

This simple idea works well when checking the blowup property and determining the blowup time. A nonuniform refinement might be more efficient in this problem since the mass is concentrated at some separated points. However this is beyond the scope of this work and is left for future study.

\subsection{A second order scheme for 1D model}

One can derive a second order scheme without much difficulty for the 1D (local or nonlocal) kinetic model. First the Strang splitting is applied. One solves the stiff source part (4.5) over a time step $\frac{\Delta t}{2}$, then solves the transport part (4.6) over $\Delta t$, then solves the stiff source part (4.5) over another $\frac{\Delta t}{2}$.

Here we describe the second order scheme for the nonlocal model in detail. It can be applied to the local model without any difficulty. The stiff part (4.5) can be solved exactly in time. Noting that the cell density $\rho$ and the chemical concentration $S$ are not changed in this step. Let

$$
\lambda=e^{-\frac{\Delta t\left(1+<\delta^{\varepsilon} S^{n}>\right)}{2 \varepsilon^{2}}},
$$

then after a time step $\Delta t / 2$,

$$
\begin{gathered}
r^{*}=\lambda r^{n}+(1-\lambda) \frac{F(v)+R\left[\delta^{\varepsilon} S^{n}\right]}{1+<\delta^{\varepsilon} S^{n}>} \rho^{n}, \\
j^{*}=\lambda j^{n}+(1-\lambda) \frac{J\left[\delta^{\varepsilon} S^{n}\right]}{1+<\delta^{\varepsilon} S^{n}>} \rho^{n}+v \partial_{x}^{(c)} \tilde{r},
\end{gathered}
$$

with

$$
\tilde{r}=\left(1-\frac{1}{\varepsilon^{2}}\right)\left(\frac{\Delta t}{2} \lambda r^{n}+\frac{F(v)+R\left[\delta^{\varepsilon} S^{n}\right]}{1+<\delta^{\varepsilon} S^{n}>} \rho^{n}\left(\frac{\varepsilon^{2}(1-\lambda)}{\left(1+<\delta^{\varepsilon} S^{n}>\right)}-\frac{\Delta t}{2} \lambda\right)\right) .
$$

Here the computation of $\delta^{\varepsilon} S^{n}$ is described in Section 4.6, $\partial_{x}^{(c)}$ is a central difference in $x$ direction.

Next a second-order TVD scheme is needed to solve the transport part over a time step $\Delta t$,

$$
\begin{aligned}
& \frac{r_{i}^{* *}-r_{i}^{*}}{\Delta t}+\frac{v}{2 \Delta x}\left(j_{i+1}^{*}-j_{i-1}^{*}\right)=\frac{v}{2 \Delta x}\left(r_{i+1}^{*}-2 r_{i}^{*}+r_{i-1}^{*}\right)-\frac{v}{4}\left(1-\frac{v \Delta t}{\Delta x}\right)\left(\sigma_{i}^{+}-\sigma_{i-1}^{+}+\sigma_{i+1}^{-}-\sigma_{i}^{-}\right), \\
& \frac{j_{i}^{* * 1}-j_{i}^{*}}{\Delta t}+\frac{v}{2 \Delta x}\left(r_{i+1}^{*}-r_{i-1}^{*}\right)=\frac{v}{2 \Delta x}\left(j_{i+1}^{*}-2 j_{i}^{*}+j_{i-1}^{*}\right)-\frac{v}{4}\left(1-\frac{v \Delta t}{\Delta x}\right)\left(\sigma_{i}^{+}-\sigma_{i-1}^{+}-\sigma_{i+1}^{-}+\sigma_{i}^{-}\right),
\end{aligned}
$$

where

$$
\sigma_{i}^{ \pm}=\frac{1}{\Delta x} \operatorname{minmod}\left(r_{i+1}^{*} \pm j_{i+1}^{*}-r_{i}^{*} \mp j_{i}^{*}, \quad r_{i}^{*} \pm j_{i}^{*}-r_{i-1}^{*} \mp j_{i-1}^{*}\right) .
$$

Finally we can update the density $\rho^{* *}$ from $r^{* *}$ and $S^{* *}$ is obtained. Then the stiff part is solved over another $\Delta t / 2$.

\subsection{The convolution}

Finally, we apply the FFT algorithm on the computation of convolution (1.3) to get $S$. The singularity of $\log |x|$ at $x=0$ makes the direct numerical integral difficult, see [8]. Noting that $\log |x|$ belongs to $L^{1}$, we can avoid this problem by taking Fourier transform first. In the numerical simulation, we will always make $f$ to be compactly supported in the computational region. It is not a problem about the periodicity of boundary condition by extending the solution to zero in a larger interval and by computing the Fourier transform there. In this way we avoid any kind of aliasing. 


\subsection{The computation of $\delta^{\varepsilon} S$}

To obtain a higher accuracy in computing $\delta^{\varepsilon} S$ in (2.1), a high order interpolation is needed to compute $S(x+\varepsilon v)$. Here we apply the FFT based interpolation,

$$
S\left(x_{j}+\varepsilon v\right)=\frac{1}{N} \sum_{k} \hat{S}_{k} e^{-i k\left(x_{j}+\varepsilon v\right)}=\frac{1}{N} \sum_{k}\left(\hat{S}_{k} e^{-i k \varepsilon v}\right) e^{-i k x_{j}},
$$

where $\hat{S}$ is the discrete Fourier transform of $S$ on grids $x_{j}$.

\section{Numerical results}

\subsection{D Nonlocal Model}

The first simulations are devoted to the one dimensional nonlocal model described in section 2.1

The following simulations are set on $x \in \Omega=[-1,1], v \in V=[-1,1]$. We take $N_{v}=64$, which can provide good enough accuracy for numerical simulations. The constant function in $V$ is chosen as the equilibrium

$$
F(v)=\frac{1}{|V|} \mathbf{1}_{V}
$$

In this setting, the critical mass of blow up for the limiting Keller-Segel system is

$$
M_{c}=2 \pi
$$

The initial conditions in the simulation are always given by,

$$
\rho^{I}(x)=C e^{-80 x^{2}}, \quad f_{\mathcal{E}}^{I}(x, v)=\rho^{I}(x) F(v),
$$

where $C=C(M)$ is a constant determined by the total mass $M$.

As predicted in [11], in the supercritical case $M>M_{c}$, the solutions to the kinetic system converge to that of the Keller-Segel system only in finite time (before blow up time). After that, the asymptotic limit is not valid anymore. To capture the behavior of solutions to the kinetic system after that time, one has to resolve the small scale $\varepsilon$. Therefore in the simulation, we need $\Delta x=O(\varepsilon)$. While in the subcritical case, as will be shown in the following sections, the asymptotic limit seems to be valid over any time period. One can take $\Delta x$ independent of $\varepsilon$, as in a typical AP scheme [21].

As for the time step length $\Delta t$, the simulation results suggest that, for the sake of stability, one needs $\Delta t=\varepsilon \Delta x /\left(2 v_{\max }\right)$ for long time simulation in the supercritical case. While in the subcritical case $M<M_{c}$, as $\varepsilon \rightarrow 0$, the diffusive nature of the Keller-Segel system requires $\Delta t=\Delta x^{2} / 2$. A general choice of $\Delta t$ would be

$$
\Delta t=\max \left\{\frac{\varepsilon \Delta x}{2 v_{\max }}, \frac{\Delta x^{2}}{2}\right\} .
$$

\subsubsection{Convergence order of numerical scheme}

In this section we test the convergence order of numerical scheme described in Section 4.4 We check the following error,

$$
e_{\Delta x}(f)=\frac{\left\|f_{\Delta x}(t)-f_{2 \Delta x}(t)\right\|_{1}}{\left\|f_{2 \Delta x}(0)\right\|_{1}}
$$




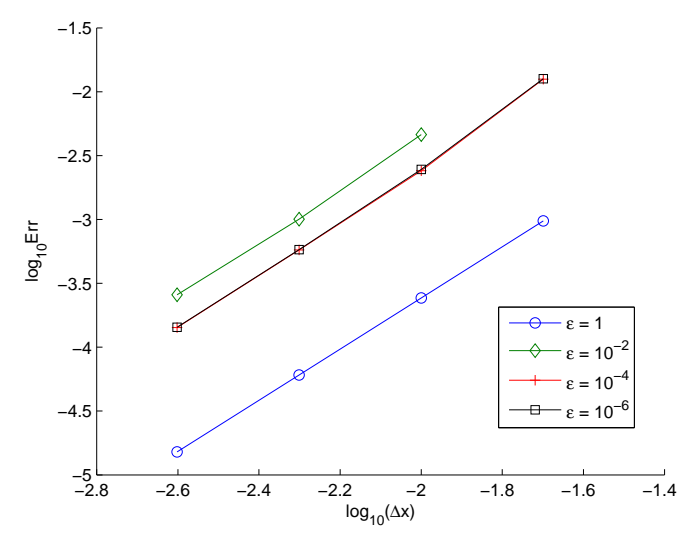

(a) $M>M_{c}$.

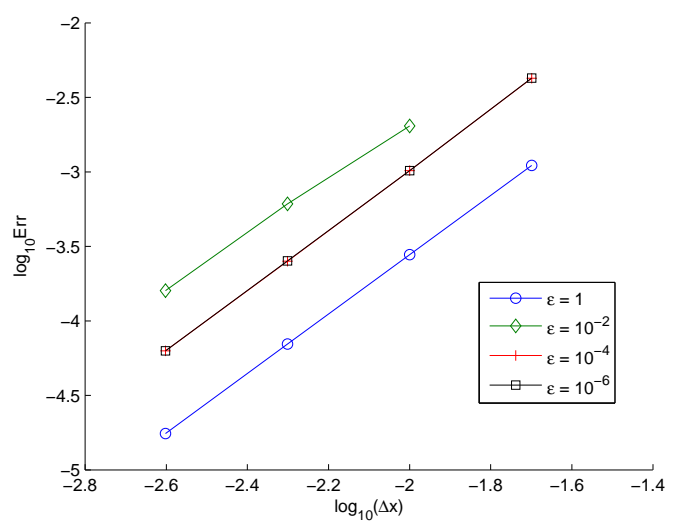

(b) $M<M_{c}$.

Figure 2: 1D nonlocal model. The convergence order of scheme described in Section 4.4 for different $\varepsilon$. Left: $M=4 \pi>M_{c}, t=0.0025<t_{b}$; Right: $M=\pi<M_{c}, t=0.025$.

This can be considered as an estimation of the relative error in $l^{1}$ norm, where $f_{h}$ is the numerical solution computed from a grid of size $\Delta x=\frac{x_{\max }-x_{\min }}{N_{x}}$. The numerical scheme is said to be $k$-th order if $e_{\Delta x} \leq C \Delta x^{k}$. The computations are performed with $N_{x}=100,200,400,800,1600,1600, \Delta t=\frac{\Delta x^{2}}{2}$.

Figure 2 (a) shows the convergence results at $t=0.0025$ for different $\varepsilon$, with initial data given by (5.1) and total mass $M=4 \pi>M_{c}$. The solution to the Keller-Segel system with total mass $4 \pi$ blows up at $t_{b} \approx 0.0039$ (see next section). The scheme shows second order convergence (in $l^{1}$ norm) for supercritical mass before blow up time.

Figure 2 (b) shows the convergence results at $t=0.025$ for different $\varepsilon$, with initial data given by (5.1) and subcritical mass $M=\pi<M_{c}$. The second order convergence (in $l^{1}$ norm) is observed for all $\varepsilon$.

In conclusion, our scheme has second order convergence, uniformly in $\varepsilon$. This is a common result for AP scheme, see [15].

The above simulations are performed with the transport equation (4.6) solved by a Lax-Wendroff method. The use of the second order TVD method described in Section 4.4 shows a lower, but still uniform convergence in $\varepsilon$.

\subsubsection{Global existence and finite time blow up}

Following the proof in [11], one can show that the solution to the kinetic system (2.3)-(2.5b) is bounded on $[0, T]$, for any time $T$. However, the Patlak-Keller-Segel system (1.4) can present a blow-up phenomenon in finite time, see [8].

Now we take the initial data (5.1) with supercritical mass $M=4 \pi>M_{c}=2 \pi$. The blowup time $t_{b} \approx$ 0.0039 from the numerical simulation.

Figure 3 shows the global boundedness of kinetic system for different $\varepsilon$ and the finite time blowup for the corresponding Keller-Segel model, by drawing the time evolution of the maximum value of $\rho_{\varepsilon}$ and $\rho$.

\subsubsection{AP property: Convergence in $\varepsilon$ at a finite time interval}

As mentioned before, it has been shown in [11] that the solution of the kinetic system can converge to that of the Patlak-Keller-Segel system weakly in a finite time interval $\left[0, t^{*}\right]$ with $t^{*}$ small enough. Here we 


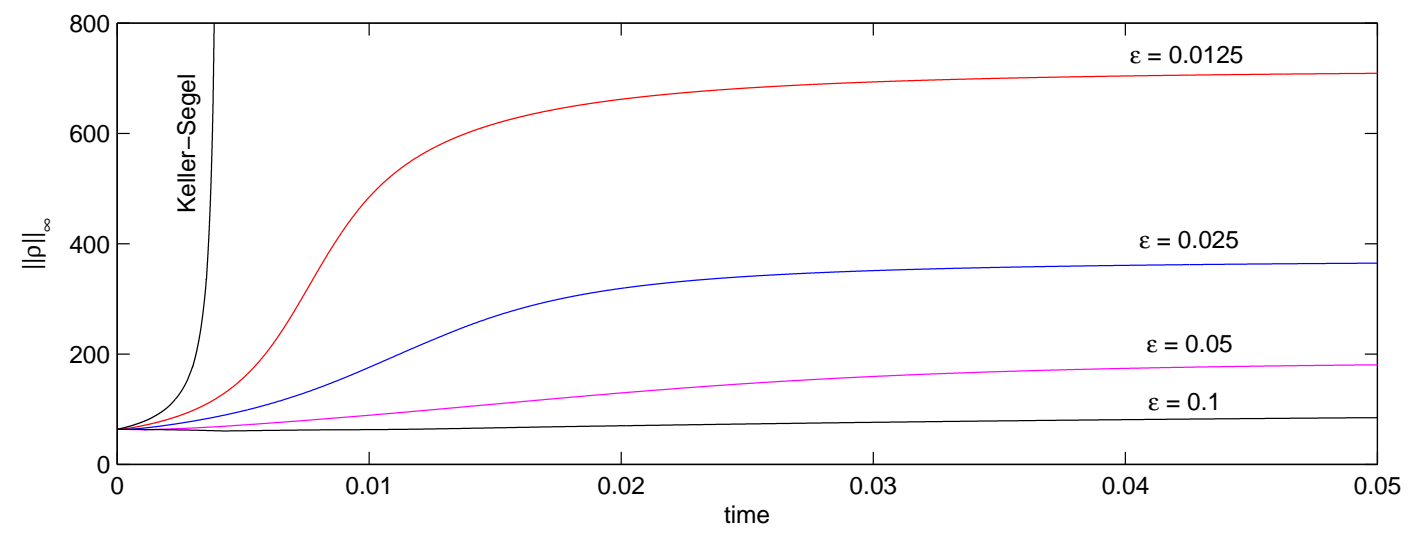

Figure 3: 1D nonlocal model. Time evolution of kinetic system and Keller-Segel model for supercritical mass $M=4 \pi$.

numerically check this convergence. We use the same grid size $\Delta x=\frac{1}{2000}$ for different $\varepsilon$ in this subsection.

In this section, we use the notations $\rho_{\varepsilon}$ and $f_{\varepsilon}$ to for the solutions to the kinetic system, while $\rho_{0}$ for the Keller-Segel model.

The supercritical case is studied first. We take initial data (5.1) with $M=4 \pi$. Figure 4 shows the convergence of $f_{\varepsilon} \rightarrow \rho_{0} F$ as $\varepsilon \rightarrow 0$ at time $t=0.002<t_{b}$, where $t_{b} \approx 0.0039$ is the blow up time of the limiting Keller-Segel model. Figure 4 (a) sketches the shape of $\rho_{\varepsilon}(x)=\int_{V} f(x, v) \mathrm{d} v$. As $\varepsilon \rightarrow 0$, $\rho_{\varepsilon}$ (solutions to kinetic system) approach to $\rho_{0}$, the solution of the limiting Keller-Segel system. Figure 4(b) shows that the convergence order of $f_{\varepsilon} \rightarrow \rho_{\varepsilon} F$ is almost first order (around 0.85) in $l^{2}$ norm.

For a subcritical case, the initial data are taken to be (5.1) with $M=\pi$. After a relatively long time $t=0.01$, we obtain the very similar results. See figure 5 . Now we have first order convergence in $\varepsilon$, in $l^{2}$ norm.

\subsubsection{The stationary solution of kinetic system}

In this subsection we will study the solution of kinetic system with large initial mass $M=4 \pi$ at a relatively long time $t=0.1$. After a long time, the solution stabilizes toward a stationary state. This has not been proved nor intuitively discussed in the literature. Figure 6 shows the function $\varepsilon \rho_{\varepsilon}(\varepsilon x)$ at time $t=0.1$. Figure 7 shows the function $\frac{f(\varepsilon x, v)}{\rho(\varepsilon x)}$ as a function of $v$ at different $x$. These two figures suggest the stationary state satisfies

$$
f_{\infty}^{\varepsilon}(x, v)=\frac{1}{\varepsilon} \tilde{\rho}_{\infty}\left(\frac{x}{\varepsilon}\right) \tilde{F}_{\infty}\left(\frac{x}{\varepsilon}, v\right) .
$$

for some functions $\tilde{\rho}_{\infty}(x)$ and $\tilde{F}_{\infty}(x, v)$. Therefore, let us consider the ansatz

$$
\begin{aligned}
\tilde{f}(t, x, v) & =\varepsilon f_{\varepsilon}(t, \varepsilon x, v) \\
\tilde{\rho}(t, x) & =\varepsilon \rho_{\varepsilon}(t, \varepsilon x) \\
\tilde{S}(t, x) & =-\frac{1}{\pi} \log |x| * \rho=S_{\varepsilon}(\varepsilon x)+C_{\varepsilon}
\end{aligned}
$$




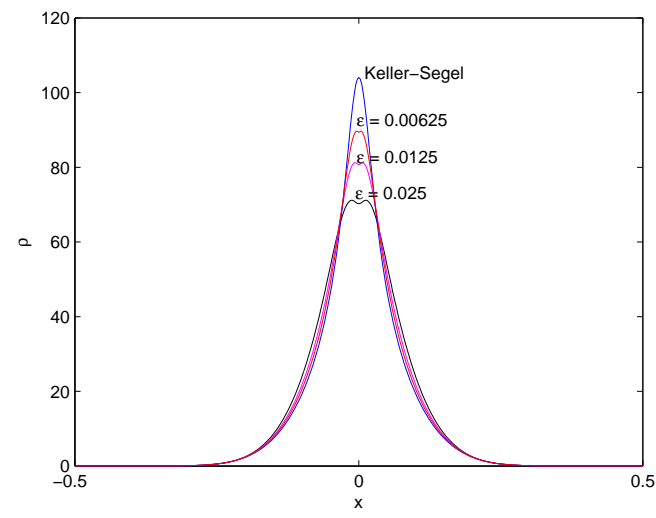

(a) $\rho_{\varepsilon}(x)$.

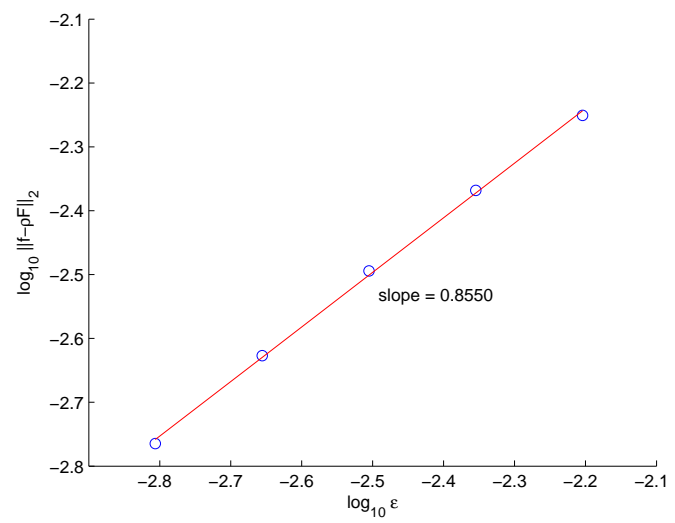

(b) Convergence in $\varepsilon$.

Figure 4: 1D nonlocal model. The solutions of kinetic system and Keller-Segel system at time $t=0.002$, before blow up time. The super-critical mass $M=4 \pi$ is used. The left figure shows $\rho_{\varepsilon}$ (for different $\varepsilon$ ) and $\rho$. The right figure gives the convergence order of $\left\|f_{\varepsilon}(x, v)-\rho_{\varepsilon}(x) F(v)\right\|_{2}$.

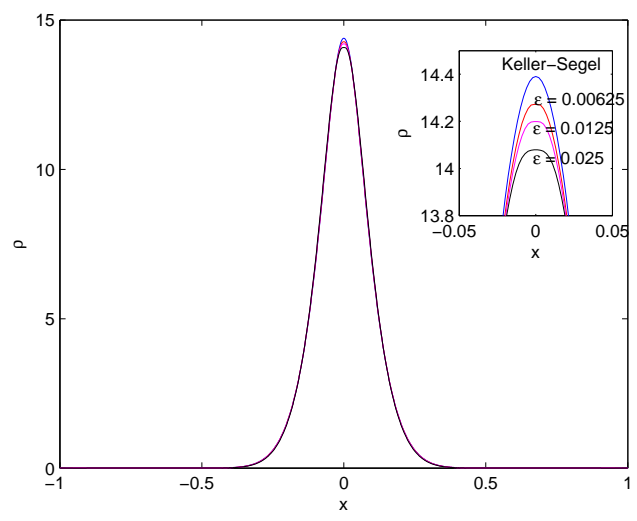

(a) $\rho_{\varepsilon}(x)$.

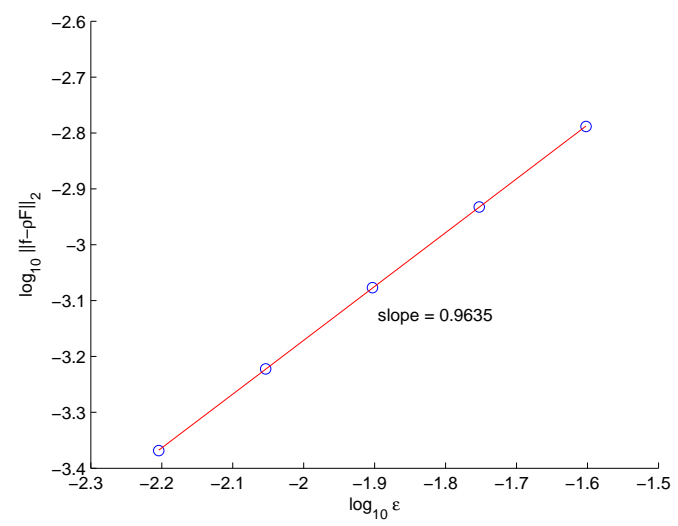

(b) Convergence in $\varepsilon$.

Figure 5: 1D nonlocal model. The solutions of kinetic system and Keller-Segel system at time $t=0.01$, before blow up time. The super-critical mass $M=4 \pi$ is used. The left figure shows $\rho_{\varepsilon}$ (for different $\varepsilon$ ) and $\rho$. The right figure gives the convergence order of $\left\|f_{\varepsilon}(x, v)-\rho_{\varepsilon}(x) F(v)\right\|_{2}$. 


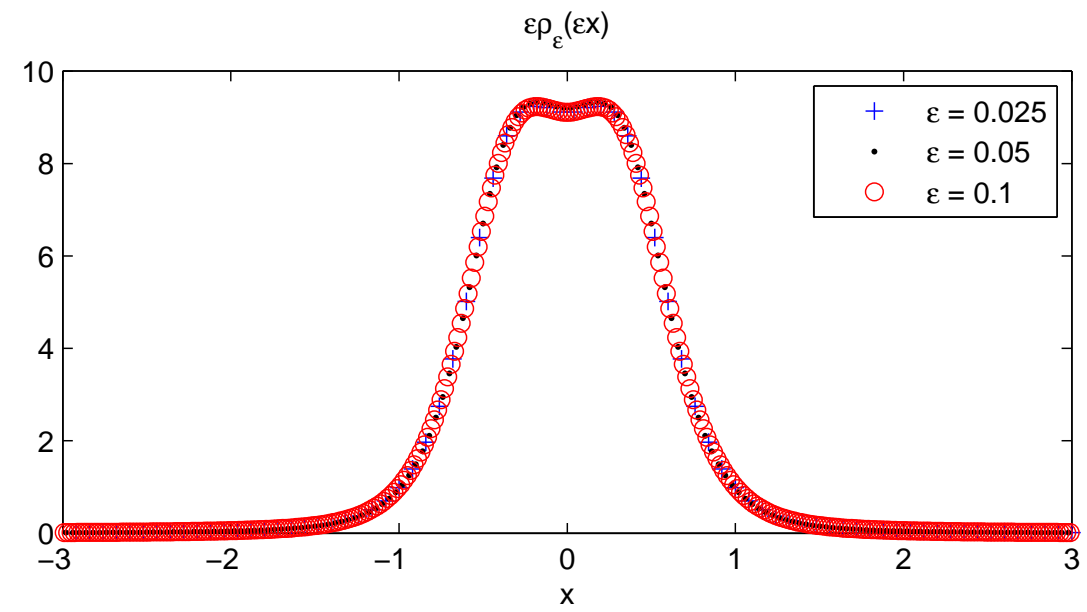

Figure 6: 1D nonlocal model. The function $\varepsilon \rho_{\varepsilon}(\varepsilon x)$ for different $\varepsilon$. For supercritical mass $M=4 \pi$ at time $t=0.1 \gg t_{b}$.

where $C_{\varepsilon}=\frac{M}{\pi} \log \varepsilon$, with $M$ the total mass. The rescaled variables satisfy the following equations

$$
\begin{gathered}
\varepsilon^{2} \frac{\partial \tilde{f}}{\partial t}+v \frac{\partial \tilde{f}}{\partial x}=(F(v)+\delta \tilde{S}(x, v)) \tilde{\rho}-\left(1+\int \delta \tilde{S}\left(x, v^{\prime}\right) \mathrm{d} v^{\prime}\right) \tilde{f} \\
\tilde{S}=-\frac{1}{\pi} \log |x| * \tilde{\rho}
\end{gathered}
$$

where $t>0, x \in \Omega_{\varepsilon}=\left[-\frac{x_{\max }}{\varepsilon}, \frac{x_{\max }}{\varepsilon}\right], v \in V=\left[-v_{\max }, v_{\max }\right]$. As $t \rightarrow \infty, \frac{\partial \tilde{f}}{\partial t} \rightarrow 0$, we have the stationary solution $f_{\infty}^{\mathcal{E}}$ should solve

$$
v \frac{\partial f_{\infty}^{\mathcal{E}}}{\partial x}=\left(F(v)+\delta \tilde{S}_{\infty}(x, v)\right) \tilde{\rho}_{\infty}-\left(1+\int \delta \tilde{S}_{\infty}\left(x, v^{\prime}\right) \mathrm{d} v^{\prime}\right) \tilde{f}
$$

where $\tilde{S}_{\infty}$ is obtained from $\tilde{\rho}_{\infty}$. Clearly $\tilde{F}(x, v)$ satisfies

$$
\int_{V} \tilde{F}(x, v) \mathrm{d} v=1 \quad \text { and } \quad \int_{V} v \tilde{F}(x, v) \mathrm{d} v=0 .
$$

The second identity can be derived by integrating (5.4) over velocity space. These two identities are also verified numerically. $\tilde{\rho}(x)=\varepsilon \rho(\varepsilon x)$ is shown in Figure 6. Some snapshots of $\tilde{F}(x, v)$ are shown in Figure 7.

\subsubsection{The interaction between several peaks}

It has been shown the interaction between several peaks for the modified Keller-Segel system (1.4) in [3] by means of optimal transportation methods. Here we will check this interaction for the kinetic system.

Case I: Two symmetric peaks, without enough mass in each peak.- The initial condition is taken as the sum of two gaussian-like peaks,

$$
f_{\mathcal{\varepsilon}}^{I}(x, v)=C\left(\frac{1}{2} e^{-80(x-0.3)^{2}}+\frac{1}{2} e^{-80(x+0.3)^{2}}\right) F(v)
$$



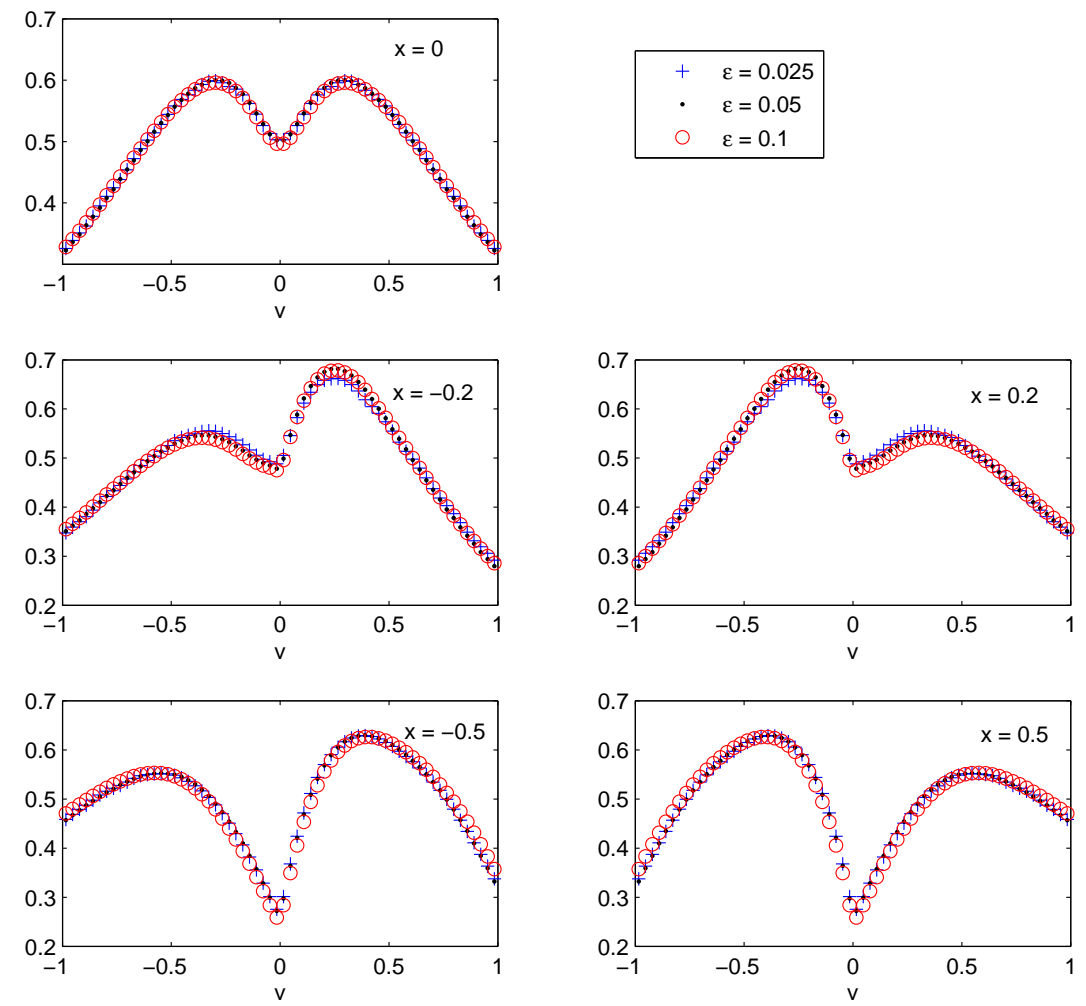

Figure 7: 1D nonlocal model. The snapshots of the stationary state $\tilde{F}(x, v)=\frac{f(\varepsilon x, v)}{\rho(\varepsilon x)}$ at certain locations $x$. For supercritical mass $M=4 \pi$ at time $t=0.1 \gg t_{b}$. 

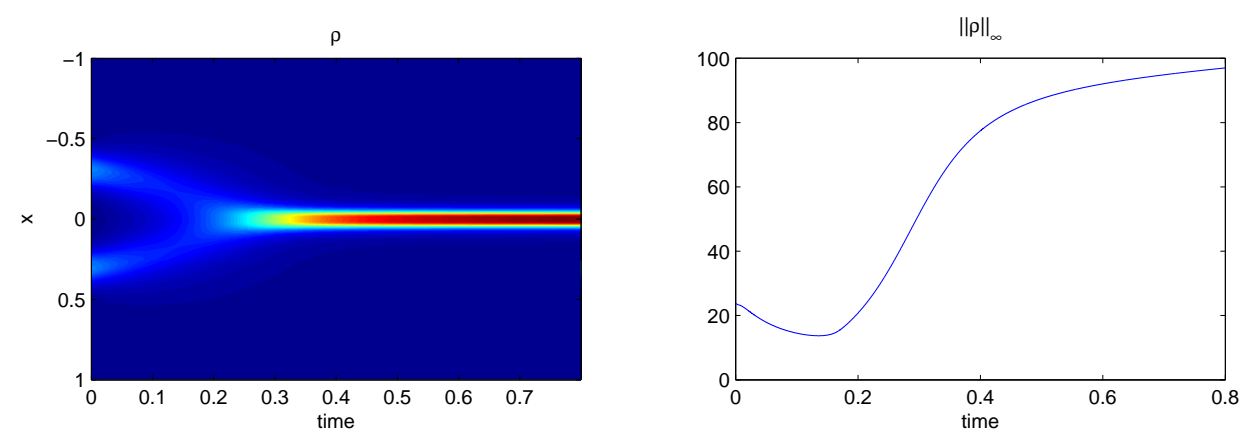

Figure 8: $1 \mathrm{D}$ nonlocal model. The attraction between peaks. $\varepsilon=0.1$. The total mass is $3 \pi$. The critical mass is $2 \pi . N x=400$.
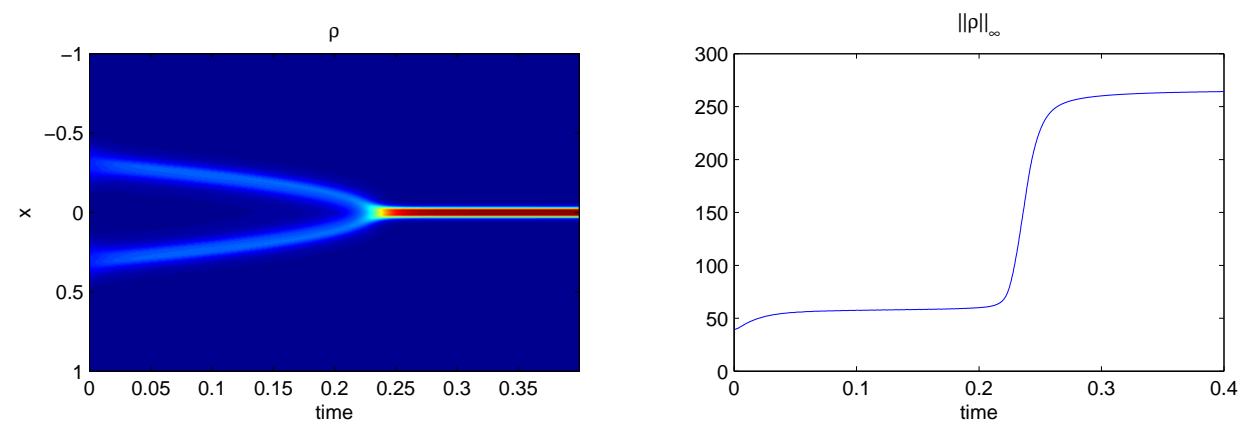

Figure 9: $1 \mathrm{D}$ nonlocal model. The attraction between peaks. $\varepsilon=0.05$. The total mass is $5 \pi$. The critical mass is $2 \pi . N x=400$.

with a suitable constant $C$ such that the total mass is $3 \pi$. We take $\varepsilon=0.1$. Figure 8 shows the time evolution of $\rho_{\varepsilon}$ (left) and $\left\|\rho_{\varepsilon}\right\|_{\infty}$ (right). The solution starts with diffusion separately, and then the peaks merge. At beginning there is no enough mass in either peak to concentrate $\left(\frac{3 \pi}{2}<M_{c}=2 \pi\right)$. But after they merge, the total mass in the new peak is large enough to form an aggregation.

Case II: Two symmetric peaks, with enough mass in each peak.- We take the same initial setting (5.5), with a different constant $C$ such that the total mass is $5 \pi$. Now the mass in each peak is large enough to concentrate $\left(\frac{5 \pi}{2}>M_{c}=2 \pi\right)$. In Figure 9 we can see two distinguished different phases during the time evolution. The first phase consists in the appearance of a metastable state where the concentration in each peak is formed but slowly moving toward each other. Then the two peaks merge, which increases the total mass into the final larger peak. It continues to aggregate the mass around it and finally reaches the stationary state.

Case III: Two asymmetric peaks, with enough mass in each peak.- We take a nonsymmetric initial setting

$$
f_{\mathcal{E}}^{I}(x, v)=C\left(2.2 e^{-80(x-0.3)^{2}}+2.8 e^{-80(x+0.3)^{2}}\right) F(v)
$$

with a suitable constant $C$ such that the total mass is $5 \pi$. The mass in each peak is still large enough to concentrate and we see that the mass tends to concentrate around the center of mass located this time much closer to the first peak due to asymmetry in Figure 10 . 

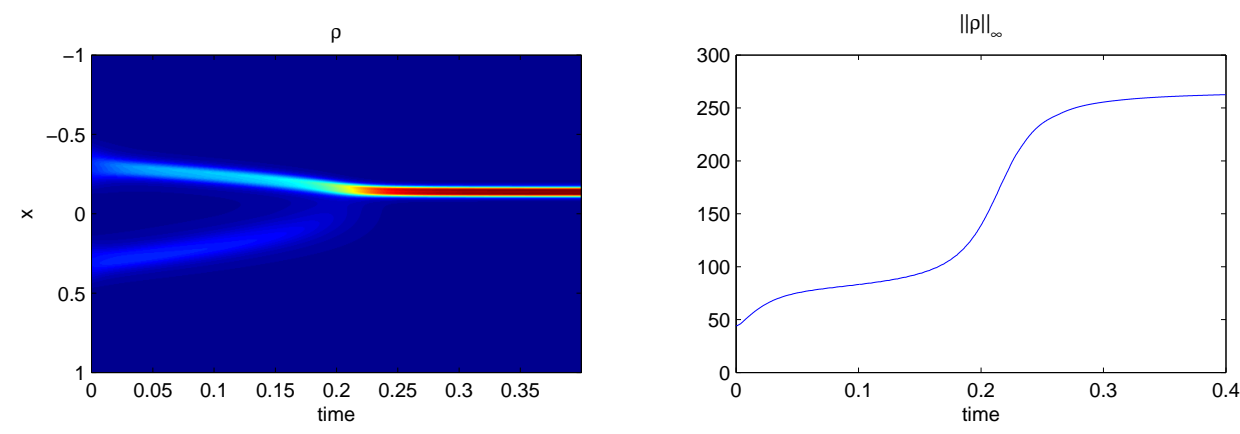

Figure 10: 1D nonlocal model. The attraction between peaks. $\varepsilon=0.05$. The total mass is $5 \pi$ with $2.2 \pi$ near $x=0.3,2.8 \pi$ near $x=-0.3$. The critical mass is $2 \pi$. $N x=400$.
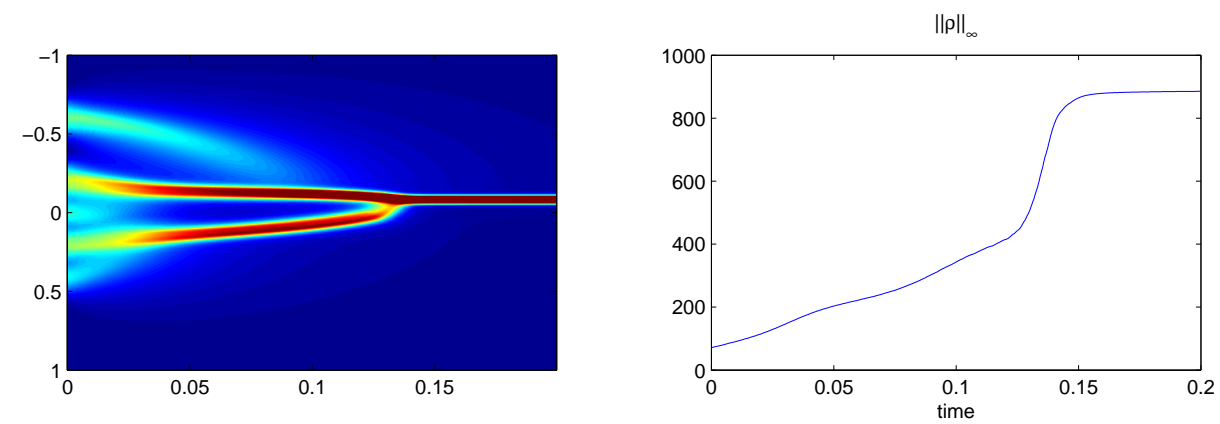

Figure 11: 1D nonlocal model. The interaction between five peaks. $\varepsilon=0.05$. The total mass is $11 \pi$. The critical mass is $2 \pi$. $N x=400$.

Case IV: Five unsymmetric peaks, with total mass $M>5 M_{c^{\circ}}$ - We take a nonsymmetric initial setting, with five different peaks,

$$
f_{\mathcal{E}}^{I}(x, v)=C \sum_{j=1}^{5} w_{j} e^{-160\left(x-c_{j}\right)^{2}}
$$

with $w=[0.5,1.2,0.8,0.6,1], c=[0.4,-0.2,-0.6,0,0.2]$. Here we pick up a suitable constant $C$ such that the total mass is $11 \pi$. Now, we observe in Figure 11 the complicated dynamics of merging between the different peaks before forming the final peak at the center of mass and converging toward the stationary state.

\subsection{The 1D local model: blow up in finite time}

We numerically check the open problem of the blow up property of the solution to the one dimensional kinetic local model (2.7). As mentioned before, a theoretical prediction on this blow up is still lacking, see [31]. We consider the initial data given by (5.1). The critical mass for corresponding Keller-Segel model is again

$$
M_{c}=2 \pi
$$

For the super-critical case, we take total mass $M=5 \pi$. 


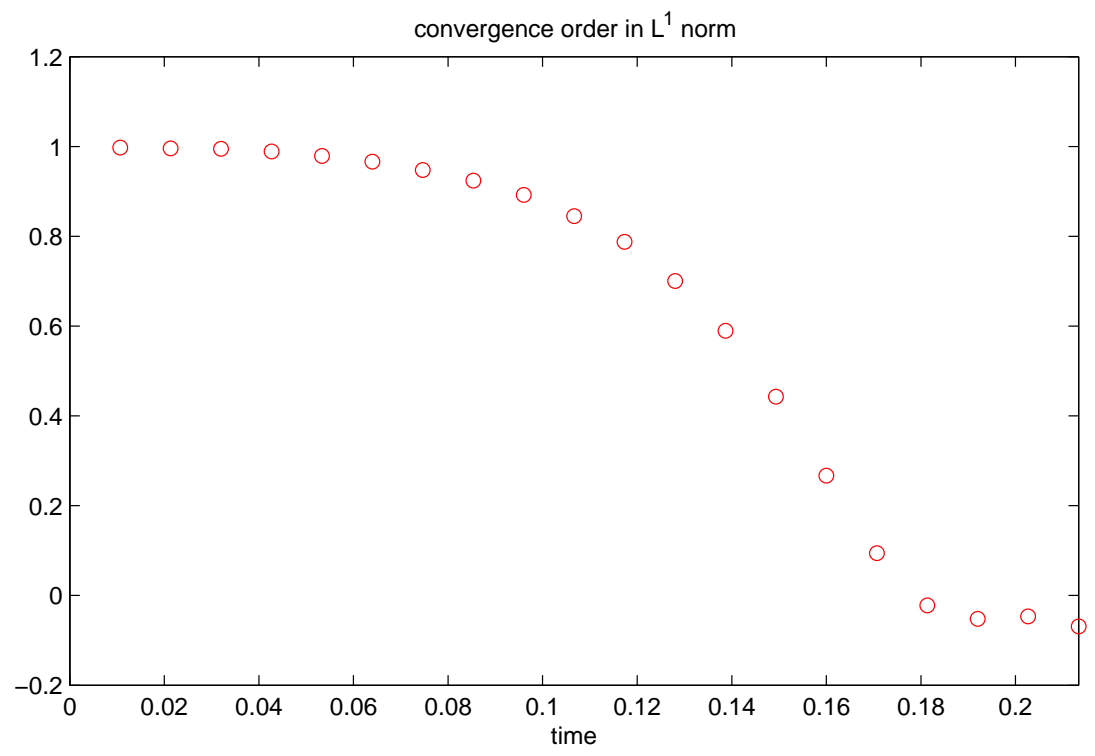

Figure 12: 1D local model. The convergence order of the solutions to 2.7) at different time. $\varepsilon=0.4$. The total mass is $5 \pi>M_{c}$.

\subsubsection{The problem in the convergence with fixed grids}

In this test $\varepsilon=0.4$. Different grid sizes in $x$ are used. The number of grid points in each of the simulations are $N_{x}=250,500,1000,2000,4000,8000$ respectively and the time step sizes are taken as $\Delta t=\varepsilon \Delta x / v_{\max }$. We still consider the convergence order defined as in (5.2).

Figure 12 shows the convergence order in $L^{1}$ norm at different times $\tau_{k}=k t_{\max } / 20$, where $1 \leq k \leq 20$. The solutions show a first order convergence when $t<0.12$, then the convergence order decreases as time evolves. After $t>0.16$ a negative convergence order is seen, which means the scheme is not convergent after that time. One cannot improve these convergence orders by using a finer grid. Figure 12 strongly suggests that the blowup happens in the solution during $0.12<t<0.16$. Next we investigate this time period by using the adaptive grids proposed in section 4.3

\subsubsection{The convergence with adaptive grids}

Now we use the adaptive grids proposed in section 4.3. We start the simulation with different grid sizes $N_{x}=500,1000,2000,4000$. Figure 13 shows the time evolution of $\|\rho\|_{\infty}$. A nice convergence toward some density blow-up is observed.

\subsubsection{The convergence as $\varepsilon \rightarrow 0$}

Next we study the convergence as $\varepsilon \rightarrow 0$. We apply the adaptive grids described above and take $N_{x}=1000$ at beginning. We take total mass to be $M=5 \pi>M_{c}$. Since the solutions to kinetic equations also blow up in finite time, they converge to the solution of Keller-Segel system in a totally different way. Figure 14 shows the time evolution of $\|\rho\|_{\infty}$ of kinetic equations with different $\varepsilon$, as well as that Keller-Segel system. The way of asymptotic convergence is totally different with Figure 3, where the solutions of kinetic equations are globally bounded. 


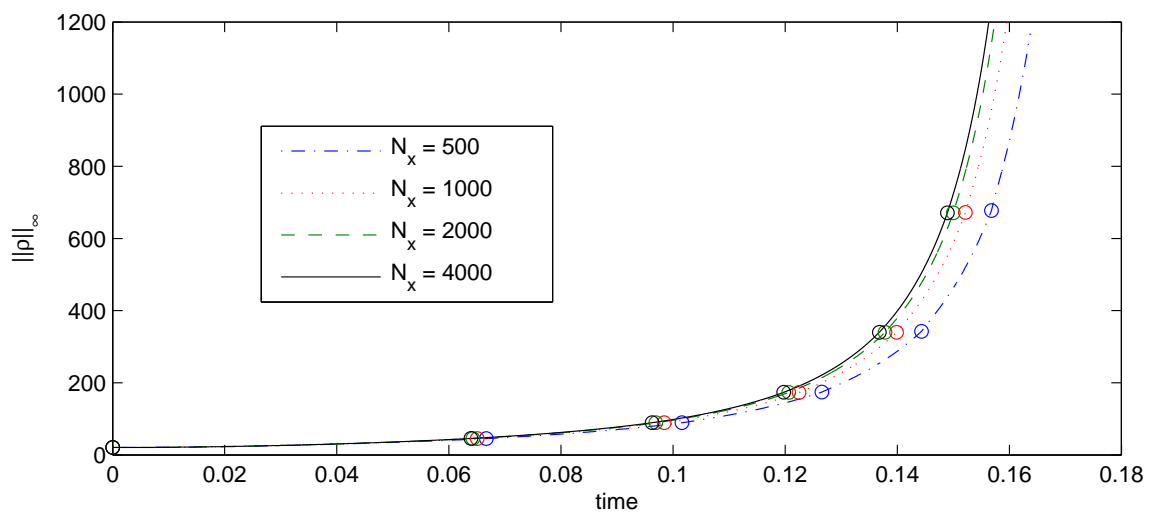

Figure 13: 1D local model. The time evolution of $\|\rho\|_{\infty}$ by using adaptive grids, with different initial grids. The circles shows the time steps when the grids are doubled. $\varepsilon=0.4$. The total mass is $5 \pi>M_{c}$.

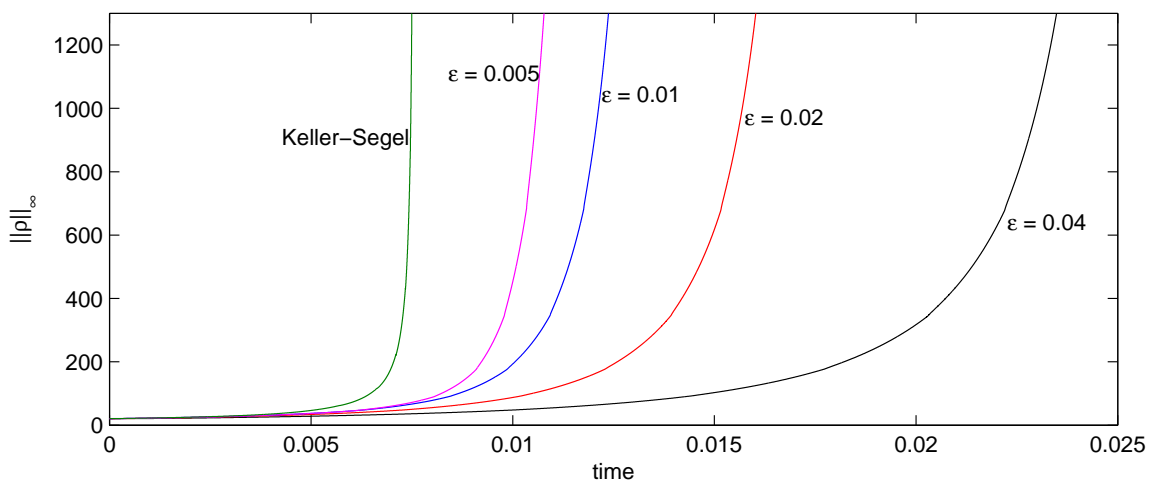

Figure 14: 1D local model. The time evolution of $\|\rho\|_{\infty}$ by using adaptive grids, with different $\varepsilon$. The total mass is $5 \pi>M_{c}$. Initially $N_{x}=1000$. 


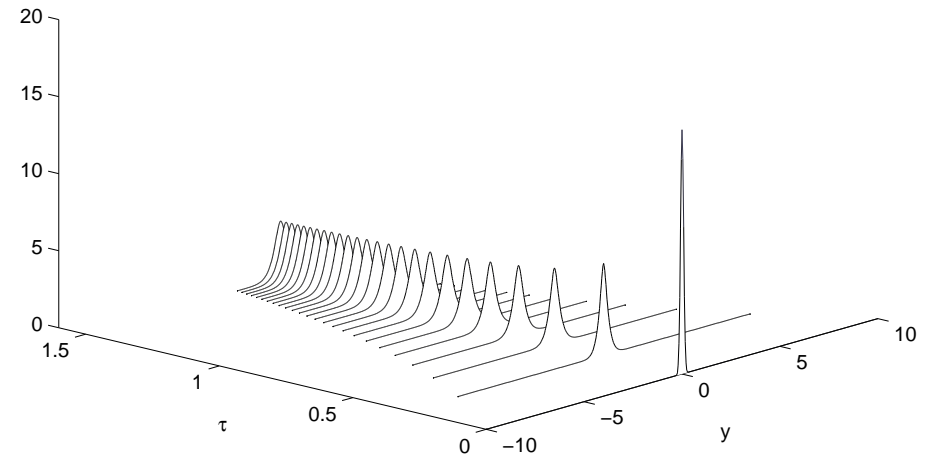

(a) $\tilde{\rho}(\tau, y)$.

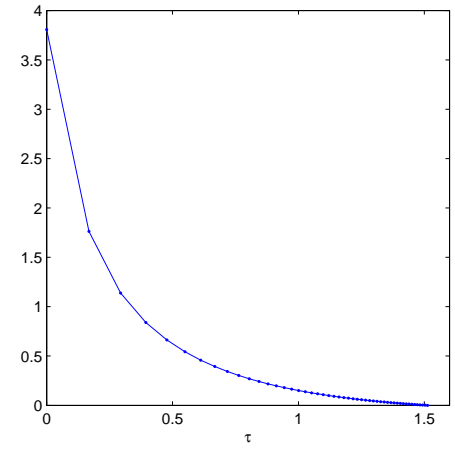

(b) $\left\|\tilde{\rho}(\tau, y)-\tilde{\rho}_{\infty}\right\|_{1}$.

Figure 15: 1D local model. The time evolution of $\tilde{\rho}$ for the subcritical mass $M=\pi<M_{c} . N_{x}=2000$. $\varepsilon=0.2$.

\subsubsection{Subcritical case: long time behavior}

Now we check the long time behavior of subcritical case. Let us remark that in the case of the limiting Keller-Segel model, it is known that the long time asymptotics should be given by a self-similar solution whose profile is dictated by a stationary scaled problem, see [3] for instance. We now check this point for the 1D local kinetic model by taking as total mass $M=\pi$. The simulation is performed on $x \in[-10,10]$. A fixed grid with $N_{x}=2000$ is used, with $\varepsilon=0.2$. We consider the same change of variables as for the Keller-Segel model,

$$
\begin{gathered}
R(t)=\sqrt{1+2 t}, \quad y=\frac{x}{R(t)}, \quad \tau=\log R(t), \\
\rho(t, x)=\frac{1}{R(t)} \tilde{\rho}(\tau, y) .
\end{gathered}
$$

Here $\rho$ is the density we computed from numerical simulation.

Figure 15 a) shows the time $(\tau)$ evolution of $\tilde{\rho}$ until time $\tau=1.5(t=10)$ as a function of $y$. It strongly suggests that $\tilde{\rho}$ is approaching some stationary state. We denote it by $\tilde{\rho}_{\infty}$. In Figure 15 (b) we show the time evolution of the relative error $\left\|\tilde{\rho}(\tau, y)-\tilde{\rho}_{\infty}\right\|_{1}$, with $\tilde{\rho}_{\infty}$ approximated through (5.6) at time $\tau=10$. Clearly the solutions are approaching the stationary state, in other words,

$$
\left\|\rho(t, x)-\frac{1}{R(t)} \tilde{\rho}_{\infty}\left(\frac{x}{R(t)}\right)\right\|_{1} \rightarrow 0, \quad \text { as } t \rightarrow \infty .
$$

\subsection{The 2D local model: solution behavior between theoretical thresholds}

In the final simulation we test the 2D local model. As shown in [7], the solution blows up with total mass larger than the critical mass

$$
M_{c}=\frac{32 \pi}{|V|}=32
$$


where $|V|=\pi \omega_{\max }^{2}=\pi$. And the global solution exists with the total mass lower than the other threshold,

$$
m_{c}=\frac{0.806 \pi}{|V|}=0.806
$$

Besides, as mentioned in the introduction, the critical mass for the corresponding Keller-Segel model (3.3) is

$$
M_{K S}=\frac{8 \pi D}{\chi}=16
$$

The gap between the two estimates $M_{c}$ and $m_{c}$ should be much smaller as observed from our numerical simulation. It is difficult to ascertain based on numerical simulations if the kinetic system show a clear dichotomy as in the Keller-Segel system.

We take $r_{\max }=2, \omega_{\max }=1$, with the equilibrium given by

$$
F(\omega)=\frac{1}{2 \pi \int_{0}^{\omega_{\max }} \omega \mathrm{d} \omega}=\frac{1}{\pi}
$$

The grid points in each directions are $N_{r}=1000, N_{\omega}=32, N_{\theta}=32$. The initial data are taken as

$$
\tilde{\rho}^{I}(r)=C r e^{-15 r^{2}}, \quad h^{I}(r, \omega, \theta)=\tilde{\rho}^{I}(r) F(\omega),
$$

where $C=C(M)$ is a constant determined by the total mass $M$.

Figure 16] shows the time evolution of $\|\rho\|_{\infty} / M$, for different total masses $M$. We take $\varepsilon=1$. It suggests that the global solutions exists for $M \leq 17$, which is much bigger than the theoretical thresholds $m_{c}$. While for mass $M \geq 25$, the solution blows up, even if the total mass belongs to the range of masses in which there are no theoretical results [7]. Let us comment that $\|\rho\|_{\infty}$ has a upper bound due to the limitation of grid size. When we use a finer grid, this upper bound would be larger.

Finally we compute the convergence of the solutions to the kinetic system as $\Delta r \rightarrow 0$, with different total masses $M$. As shown in Figure 17, the numerical scheme shows a fist order convergence for $M \leq 15$ (note that the critical mass of the Keller-Segel model is $M_{K S}=16$ ). While for $M \geq 25$, the numerical solutions do not convergence, which suggests that the solutions blow up.

\section{Acknowledgements}

This work was partially supported by NSF grant No. DMS-0608720, NSF FRG grant DMS-0757285. JAC was partially supported by the projects Ministerio de Ciencia e Innovación MTM2011-27739-C04-02 and 2009-SGR-345 from Agència de Gestió d'Ajuts Universitaris i de Recerca-Generalitat de Catalunya. BY would like to thank Professor Shi Jin in University of Wisconsin-Madison for the guidance and fruitful discussion. BY also would like to thank the warm hospitality at the Department of Mathematics in Universitat Autònoma de Barcelona during his visit. Both authors would like to thank IPAM where part of this work was initiated during the kinetic theory program.

\section{References}

[1] W. Alt. Biased random walk models for chemotaxis and related diffusion approximations. Journal of Mathematical Biology, 9:147-177, 1980. 10.1007/BF00275919. 


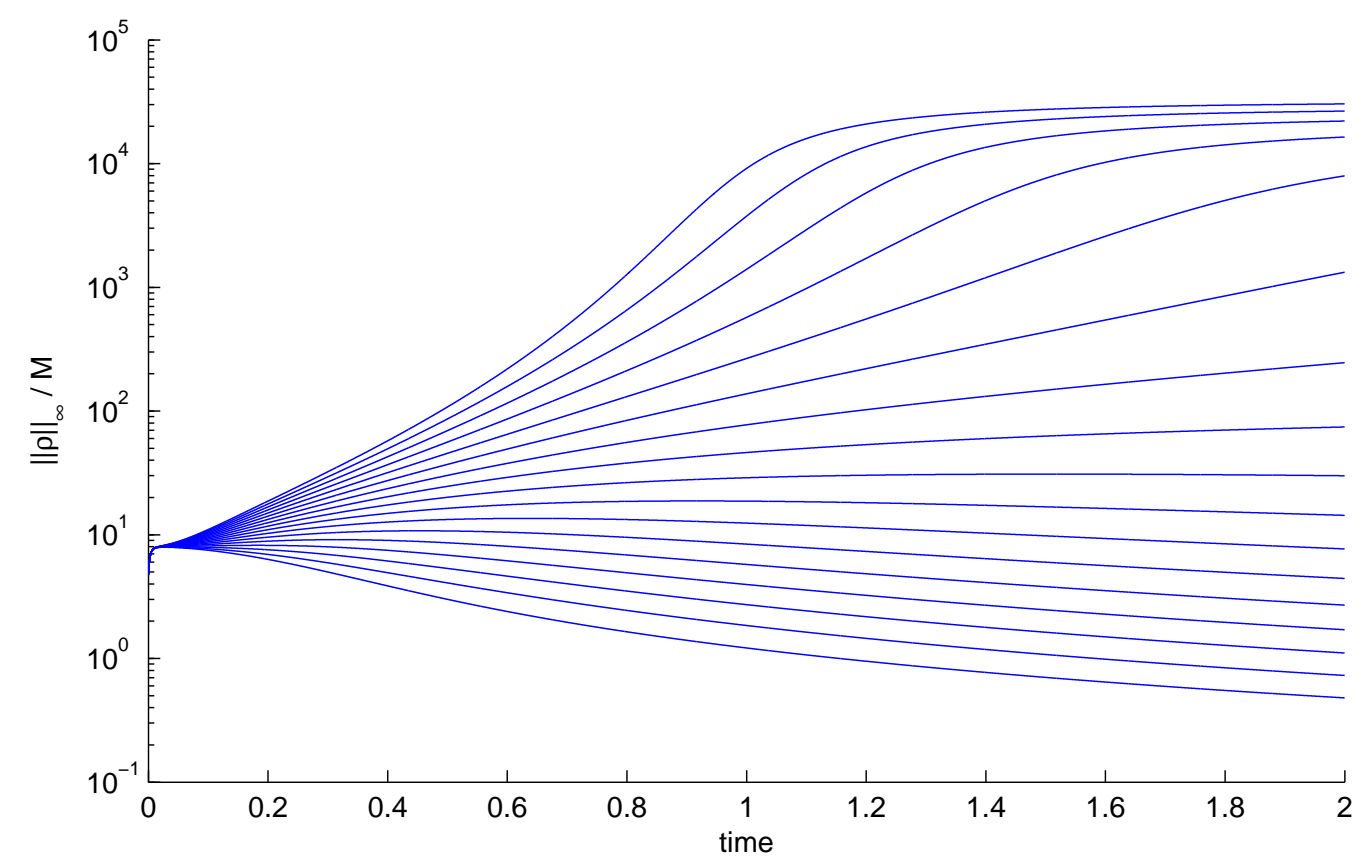

Figure 16: 2D local model. The time evolution of $\frac{\|\rho\|_{\infty}}{M}$ for the 2D local model with total masses M. From up to down: $M=33,31,29, \ldots, 3,1 . N_{r}=1000 . \varepsilon=1$.

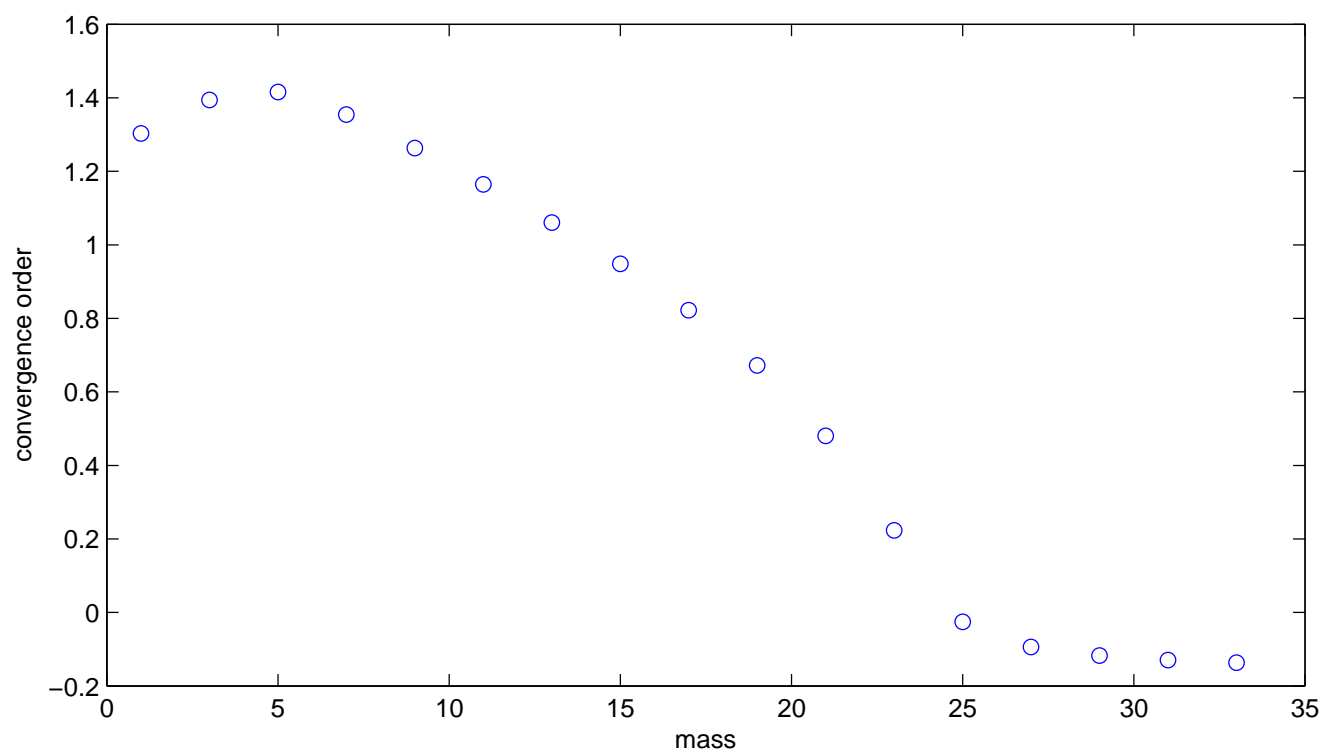

Figure 17: 2D local model. The convergence order (in $L^{1}$ norm) of the solutions to the 2D kinetic system at time $t=2$ as $\Delta r \rightarrow 0$, with different total masses $M . \varepsilon=1$. 
[2] W. Alt. Singular perturbation of differential integral equations describing biased random walks. $J$. Reine Angew., 322:15-41, 1981.

[3] A. Blanchet, V. Calvez, and J. A. Carrillo. Convergence of the mass-transport steepest descent scheme for the subcritical Patlak-Keller-Segel model. SIAM Journal on Numerical Analysis, 46(2):691-721, 2008.

[4] A. Blanchet, E. A. Carlen, and J. A. Carrillo. Functional inequalities, thick tails and asymptotics for the critical mass Patlak-Keller-Segel model. preprint arxiv: 1009.0134v1.

[5] A. Blanchet, J. A. Carrillo, and N. Masmoudi. Infinite time aggregation for the critical Patlak-KellerSegel model in $\mathbb{R}^{2}$. Comm. Pure Appl. Math., 61(10):1449-1481, 2008.

[6] A. Blanchet, J. Dolbeault, and B. Perthame. Two-dimensional Keller-Segel model: optimal critical mass and qualitative properties of the solutions. Electron. J. Differential Equations, pages No. 44, 32 pp. (electronic), 2006.

[7] N. Bournaveas and V. Calvez. Critical mass phenomenon for a chemotaxis kinetic model with spherically symmetric initial data. Annales de l'Institut Henri Poincare (C) Non Linear Analysis, In Press, Corrected Proof:-, 2009.

[8] V. Calvez, B. Perthame, and M. Sharifi tabar. Modified Keller-Segel system and critical mass for the log interaction kernel. CONTEMPORARY MATHEMATICS, (429):45-62, 2007.

[9] J. A. Carrillo, I. M. Gamba, A. Majorana, and C.W. Shu. A WENO-solver for the transients of Boltzmann-Poisson system for semiconductor devices. Performance and comparisons with Monte Carlo methods. Journal of Computational Physics, 184:498-525, 2003.

[10] J. A. Carrillo, S. Hittmeir, and A. Jüngel. Cross diffusion and nonlinear diffusion preventing blow up in the Keller-Segel model. preprint, 2011.

[11] F. A. C. C. Chalub, P. A. Markowich, B. Perthame, and C. Schmeiser. Kinetic models for chemotaxis and their drift-diffusion limits. Monatshefte für Mathematik, 142(1):123-141, 2004. 10.1007/s00605004-0234-7.

[12] L. Corrias, B. Perthame, and H. Zaag. Global solutions of some chemotaxis and angiogenesis systems in high space dimensions. Milan Journal of Mathematics, 72:1-28, 2004. 10.1007/s00032-003-0026$\mathrm{x}$.

[13] J. Dolbeault and B. Perthame. Optimal critical mass in the two-dimensional Keller-Segel model in $\mathbb{R}^{2}$. C. R. Math. Acad. Sci. Paris, 339(9):611-616, 2004.

[14] J. Dolbeault and C. Schmeiser. The two-dimensional Keller-Segel model after blow-up. Discrete Contin. Dyn. Syst., 25(1):109-121, 2009.

[15] F. Golse, S. Jin, and C. D. Levermore. The convergence of numerical transfer schemes in diffusive regimes I: Discrete-ordinate method. SIAM Journal on Numerical Analysis, 36(5):pp. 1333-1369, 1999.

[16] T. Hillen and H. G. Othmer. The diffusion limit of transport equations derived from velocity-jump processes. SIAM Journal on Applied Mathematics, 61(3):751-775, 2000. 
[17] D. Horstmann. On the existence of radially symmetric blow-up solutions for the Keller-Segel model. Journal of Mathematical Biology, 44(5):463-478, 2002. 10.1007/s002850100134.

[18] H. J. Hwang, K. Kang, and A. Stevens. Drift-diffusion limits of kinetic models for chemotaxis: A generalization. Discrete and Continuous Dynamical Systems - Series B, 5(2):319 - 334, 2005.

[19] H. J. Hwang, K. Kang, and A. Stevens. Global solutions of nonlinear transport equations for chemosensitive movement. SIAM Journal on Mathematical Analysis, 36(4):1177-1199, 2005.

[20] S. Jin. Efficient asymptotic-preserving (AP) schemes for some multiscale kinetic equations. SIAM J. Sci. Comput., 21(2):441-454, 1999.

[21] S. Jin. Asymptotic preserving (AP) schemes for multiscale kinetic and hyperbolic equations: a review. Lecture Notes for Summer School on "Methods and Models of Kinetic Theory" (M\&MKT), Porto Ercole (Grosseto, Italy), June 2010. Rivista di Matematica della UniversitÃ di Parma, to appear.

[22] S. Jin and L. Pareschi. Discretization of the multiscale semiconductor Boltzmann equation by diffusive relaxation schemes. Journal of Computational Physics, 161(1):312-330, 2000.

[23] S. Jin, L. Pareschi, and G. Toscani. Uniformly accurate diffusive relaxation schemes for multiscale transport equations. SIAM Journal on Numerical Analysis, 38(3):913-936, 2001.

[24] E.F. Keller and L.A. Segel. Initiation of slide mold aggregation viewed as an instability. J. Theor. Biol, 26:399-415, 1970.

[25] E.F. Keller and L.A. Segel. Model for chemotaxis. J. Theor. Biol, 30:225-234, 1971.

[26] H. G. Othmer, S. R. Dunbar, and W. Alt. Models of dispersal in biological systems. Journal of Mathematical Biology, 26:263-298, 1988. 10.1007/BF00277392.

[27] H. G. Othmer and T. Hillen. The diffusion limit of transport equations II: Chemotaxis equations. SIAM Journal on Applied Mathematics, 62(4):1222-1250, 2002.

[28] H. G. Othmer and A. Stevens. Aggregation, blowup, and collapse: The ABC's of taxis in reinforced random walks. SIAM Journal on Applied Mathematics, 57(4):1044-1081, 1997.

[29] C. Patlak. Random walk with persistence and external bias. Bulletin of Mathematical Biology, 15:311338, 1953. 10.1007/BF02476407.

[30] B. Perthame. In Transport Equations in Biology. Birkhaeuser Verlag, Basel-Boston-Berlin, 2007.

[31] M. Sharifi tabar. One-dimensional chemotaxis kinetic model. Nonlinear Differ. Equ. Appl., (18):139172, 2011. 